\title{
Pharmacokinetics and biodistribution of extracellular vesicles administered intravenously and intranasally to Macaca nemestrina
}

\section{Authors}

Tom Driedonks ${ }^{1}$, Linglei Jiang ${ }^{1}$, Bess Carlson ${ }^{1}$, Zheng Han ${ }^{2,3}$, Guanshu Liu ${ }^{2,3}$, Suzanne E. Queen $^{1}$, Erin N. Shirk ${ }^{1}$, Olesia Gololobova ${ }^{1}$, Lyle Nyberg ${ }^{1}$, Gabriela Lima ${ }^{1}$, Kayla Schonvisky ${ }^{1}$, Natalie Castell ${ }^{1}$, Mitchel Stover, Selena Guerrero-Martin ${ }^{1,4}$, Riley Richardson ${ }^{1}$, Barbara Smith ${ }^{5}$, Charles P. Lai ${ }^{6}$, Jessica M. Izzi ${ }^{1}$, Eric K. Hutchinson ${ }^{1}$, Kelly A.M. Pate ${ }^{1,4}$, Kenneth W. Witwer ${ }^{1,7,8,{ }^{*}}$

1. Dept. Molecular and Comparative Pathobiology, Johns Hopkins University School of Medicine, Baltimore, MD, US

2. Russell H. Morgan Department of Radiology, Johns Hopkins University School of Medicine, Baltimore, MD, US

3. F.M. Kirby Research Center, Kennedy Krieger Institute, Baltimore, MD, US

4. Division of Comparative Medicine and Department of Biological Engineering,

Massachusetts Institute of Technology, Cambridge, MA, US

5. Dept. Cell Biology, Johns Hopkins University School of Medicine, Baltimore, MD, US

6. Institute of Atomic and Molecular Sciences, Academia Sinica, Taipei, Taiwan

7. Dept. of Neurology, Johns Hopkins University School of Medicine, Baltimore, MD, US

8. The Richman Family Precision Medicine Center of Excellence in Alzheimer's Disease, Johns Hopkins University School of Medicine, Baltimore, MD, US

${ }^{*}$ Correspondence: kwitwer1@jhmi.edu 


\begin{abstract}
Extracellular vesicles (EVs) have great potential as novel drug carriers for the treatment of various diseases. These lipid bilayer vesicles are naturally abundant in mammalian tissues and circulation, can be loaded with therapeutic small molecule drugs, (si)RNA, proteins and CRISPR/Cas9, and may be engineered for retention by specific tissues. However, many questions remain on the optimal dosing, administration route, and pharmacokinetics of EVs. Previous studies have addressed biodistribution and pharmacokinetics in rodents, but little evidence is available from larger animals. Here, we investigated the pharmacokinetics and biodistribution of Expi293F-derived EVs labelled with a highly sensitive nanoluciferase reporter (palmGRET) in a non-human primate model (Macaca nemestrina), comparing intravenous (IV) and intranasal (IN) administration over a 125-fold dose range. We report that EVs administered IV had markedly longer circulation times in plasma than previously reported in mice, and were detectable in CSF after 30-60 minutes. Already after one minute following IV administration, we observed EV uptake by PBMCs, most notably B-cells. EVs were detected in liver and spleen within one hour of IV administration. None of the IN doses resulted in readily detectable EV levels in plasma, CSF, or organs, suggesting that IN delivery of EVs in large animals including humans may require reconsideration. Furthermore, EV circulation times strongly decreased after repeated IV administration, possibly due to immune responses and with clear implications for xenogeneic EV-based therapeutics. We hope that our findings from this baseline study in macaques will help to inform future research and therapeutic development of EVs.
\end{abstract}

\title{
Keywords:
}

extracellular vesicles, biodistribution, pharmacokinetics, macaques, therapeutics, drug delivery, nanomedicine, exosomes, ectosomes, microvesicles 


\section{Introduction}

Extracellular vesicles (EVs) are nano-sized vesicles produced by most or all cell types in multicellular organisms. EVs from specific cell types may also be harnessed as a treatment of a wide range of human diseases and conditions, including cancer, inflammatory diseases and tissue damage ${ }^{1-4}$. Furthermore, EVs may be loaded with therapeutic entities such as small molecule drugs ${ }^{5}$, proteins ${ }^{6}$, (si)RNA ${ }^{7,8}$, and CRISPR/Cas9 ${ }^{9,10}$. The EV lipid bilayer protects its cargo from degradation and reduces off-target effects compared with nonencapsulated therapeutics ${ }^{11}$. Moreover, EVs may be engineered for retention by specific sites in the body, including brain, through the display of cell-specific surface motifs, usually proteins or peptides ${ }^{12-14}$. Since EVs occur naturally in the bloodstream and tissues, EV administration is thought to be safe and has shown little toxic or inflammatory effects ${ }^{15,16}$. Although EVs are thus thought to be promising novel therapeutic tools, many questions remain about dosing, administration route, and pharmacokinetics.

To date, most pre-clinical studies have addressed the biodistribution and pharmacokinetics of EVs using mouse models ${ }^{17-23}$. Most studies have reported that EVs accumulate rapidly in the liver and spleen ${ }^{18,19,21,23}$, and sometimes lung ${ }^{22}$ and kidneys ${ }^{17}$. Additionally, EVs were found to have short circulation times in mice ${ }^{17,19,21-23}$. It is not well understood how the EV administration route affects the circulation time and biodistribution of EVs. One study compared intravenous (IV), subcutaneous, and intraperitoneal administration of EVs in mice 18. Compared with IV administration, subcutaneous and intraperitoneal administration resulted in lower EV uptake in liver and spleen, and higher uptake in the gastrointestinal tract and pancreas. Another study reported that intranasal (IN) administration of EVs resulted in improved brain targeting compared with IV administration ${ }^{20}$. While these mouse studies provide invaluable information on the biodistribution and therapeutic effects of EVs, results obtained in mice may have limited translatability to human physiology ${ }^{24}$. Specific therapeutic effects of EV have been tested in sheep ${ }^{25}$, and pigs ${ }^{26}$, but pharmacokinetics studies on EVs in larger animals are scarce ${ }^{3,27}$.

Here, we investigated the pharmacokinetics of EVs in a non-human primate (NHP) model, the pig-tailed macaque (Macaca nemestrina). Large animal models allow repeated sampling from the same animal, in addition to sampling of multiple biofluids at the same time, and at volumes that cannot be obtained from small rodents. NHP are also physiologically similar to humans and are the best and in some cases only models of human disease. For example, NHP are exceptionally valuable to achieve better understanding of human immunodeficiency virus (HIV) disease progression and treatment, including assessment of HIV cure strategies. Indeed, the study reported here is a prerequisite to trials of EV-associated transcriptional activators as latency-reversal agents for human immunodeficiency virus (HIV).

We made use of two novel EV reporters: palmGRET, which is a palmitoylated EGFPNanoluciferase fusion protein ${ }^{22}$, and MemGlow 700 , a near-infrared self-quenching lipid dye ${ }^{28}$. PalmGRET enables highly sensitive detection of EVs by emission of bioluminescence in the presence of a furimazine substrate ${ }^{22}$. MemGlow dye emits fluorescence in the nearinfrared range, where autofluorescence is generally reduced. Furthermore, this dye has been previously used to track tumor EV in live zebrafish ${ }^{29}$.

We assessed how IV or IN administration of different doses of EVs affects the circulation time in plasma and CSF of pig-tailed macaques. Additionally, we measured the uptake of EVs by different subsets of peripheral blood mononuclear cells (PBMCs) shortly after administration. Furthermore, we compared the biodistribution of EVs in different organs of both macaques and mice. We found that the administration route strongly affected the pharmacokinetics and tissue distribution of EV. Repeated IV administration of EVs resulted 
bioRxiv preprint doi: https://doi.org/10.1101/2021.07.28.454192; this version posted July 29, 2021. The copyright holder for this preprint (which was not certified by peer review) is the author/funder, who has granted bioRxiv a license to display the preprint in perpetuity. It is made available under aCC-BY 4.0 International license.

in more rapid clearance from the peripheral circulation, potentially via immune-mediated effects. To our knowledge, this is the first reported study on the pharmacokinetics of EVs in macaques, which we trust will inform future studies on therapeutic applications of EVs. 


\section{Materials \& Methods}

\section{Cells and plasmids}

Expi293F cells (Thermo Fisher) were maintained in Expi293 medium (Gibco, Waltham, MA) in vented shaker flasks on a shaker platform maintained at $125 \mathrm{rpm}$ in a humidified $37^{\circ} \mathrm{C}$ incubator with $8 \% \mathrm{CO}_{2}$. The pLenti-palmGRET reporter ${ }^{22}$ was provided by C.P. Lai (Addgene 158221), and endotoxin-free plasmid DNA megapreps were prepared by Genewiz (Genewiz, South Plainfield, NJ). For each EV production batch, 3x $1 \mathrm{~L}$ shaker flasks were seeded with a total of $750 \mathrm{ml}$ of cell suspension at 3E6 cells $/ \mathrm{ml}$. Cells were transfected using the Expifectamine kit (Thermo Fisher) according to the manufacturer's instructions, with 150 $\mu \mathrm{g}$ pDNA $(0.6 \mu \mathrm{g}$ pDNA per $\mathrm{ml}$ of culture) and $480 \mu \mathrm{l}$ of Expifectamine per flask. One day after transfection, $1.2 \mathrm{ml}$ Enhancer 1 and $12 \mathrm{ml}$ Enhancer 2 were added to each flask. Cultures were harvested three days after transfection. Transfection efficiency was checked on a Nikon Eclipse TE200 fluorescent microscope, cells were counted on a hemacytometer and tested for viability by Trypan blue exclusion (Thermo Fisher). Cell densities and viability of different batches at harvest are found in Supplementary Table 1.

\section{EV separation and fluorescent labelling}

Cells were removed from conditioned medium by centrifugation at $1000 \mathrm{~g}$ for $20 \mathrm{~min}$ at $4 \mathrm{C}$. Supernatant was centrifuged again at $2000 \mathrm{~g}$ for $20 \mathrm{~min}$ and filtered through a $0.22 \mu \mathrm{m}$ bottle-top filter (Corning, NY). EVs were concentrated to $75 \mathrm{ml}$ by tangential flow filtration (TFF) using two 100 kD Vivaflow 50R cassettes (Sartorius, Goettingen, Germany) run in parallel on a Cole-Parmer Masterflex L/S peristaltic pump operated at $100 \mathrm{rpm}$. The concentrated EVs were fluorescently labelled by adding $200 \mathrm{nM}$ MemGlow 700nm dye ${ }^{28}$ (Cytoskeleton Inc., Denver, CO) and incubating at RT for $30 \mathrm{~min}$. EVs were concentrated further on Amicon 15 Ultra RC 100kD filters (Millipore Sigma, Darmstadt, Germany), spun for $20-30 \mathrm{~min}$ at $4000 \mathrm{~g}$. The concentrate was loaded onto a qEV10 70nm SEC column (Izon, Medford, MA) run with DPBS (Gibco, Waltham, MA), after discarding the void volume, 5-ml fractions were collected. EV-enriched fractions 1-4 were pooled together and were concentrated again on Amicon 15 Ultra RC 100kD filters, spun for $20-30$ min at $4000 \mathrm{~g}$. EVs were aliquoted and stored in LoBind tubes (Eppendorf, Bochum, Germany) at $-80^{\circ} \mathrm{C}$.

\section{Immunoblotting}

Transfected Expi293F cell pellets were lysed in PBS + 1\% Triton-X100 and Complete protease inhibitor tablets (Roche, Mannheim, Germany) for 15 minutes on ice. Nuclei were spun down for 15 minutes at $14,000 \mathrm{rpm}$ in a tabletop centrifuge at $4^{\circ} \mathrm{C}$. Cell lysate, final EV isolate and individual SEC fractions were mixed with 4x TGX sample buffer (Bio-Rad, Hercules, CA) under non-reducing conditions, boiled for 5 minutes at $100^{\circ} \mathrm{C}$, and subjected to PAGE gel electrophoresis on a 4\%-15\% Criterion TGX Stain-Free Precast gel (Bio-Rad). Proteins were transferred to a PVDF membrane using the iBlot2 system (Thermo Fisher) run on program p0. After $1 \mathrm{~h}$ of blocking in 5\% Blotting-Grade Blocker (Bio-Rad) in PBS $+0.05 \%$ Tween-20 (PBS-T), blots were incubated overnight at $4^{\circ} \mathrm{C}$ with the following primary antibodies in blocking buffer: Rabbit-anti-Calnexin (1: 1000, ab22595, Abcam), mouse-antiCD63 (1 : 3000, \#556016, BD Biosciences), mouse-anti-CD9 (1 : 3000, \#312102,

BioLegend), rabbit-anti-Tsg101 (1:2000, ab125011, Abcam). Blots were washed 3x with PBS-T and incubated for $1 \mathrm{~h}$ at room temperature with secondary antibodies, mouse-lgGkBP-HRP (sc-516102, SantaCruz), mouse-anti-rabbit-lgG-HRP (sc-2357, SantaCruz) diluted 1 : 10,000 in blocking buffer. After washing $3 x$ with PBS-T and $2 x$ with PBS, SuperSignal West Pico PLUS Chemiluminescent Substrate (Pierce, Rockford, IL) was used for detection on an iBright FL1000 (Thermo Fisher) in chemiluminescence mode. 


\section{Nanoparticle tracking analysis}

ZetaView QUATT-NTA Nanoparticle Tracking Video Microscope PMX-420 and BASIC NTANanoparticle Tracking Video Microscope PMX-120 (ParticleMetrix, Inning am Ammersee, Germany) were used for particle quantification in scatter mode. The system was calibrated with $100 \mathrm{~nm}$ PS beads, diluted $1: 250,000$ before each run. Capture settings were sensitivity 75 , shutter 100 , minimum trace length 15 , cell temperature was maintained at $25^{\circ} \mathrm{C}$ for all measurements. Samples were diluted in $0.22 \mu \mathrm{m}$ filtered PBS to a final volume of $1 \mathrm{ml}$. Samples were measured by scanning 11 positions twice, recording at 30 frames per second. Between samples, the system was washed with PBS until no particles remained. ZetaView Software 8.5.10 was used to analyze the recorded videos with the following settings: minimum brightness 20 , maximum brightness 255 , minimum area 5 , and maximum area 1000.

\section{Transmission electron microscopy}

$10 \mu \mathrm{L}$ sample was adsorbed to glow-discharged carbon-coated 400 mesh copper grids by flotation for 2 minutes. Grids were quickly blotted and rinsed by flotation on 3 drops (1 minute each) of Tris-buffered saline. Grids were negatively stained in 2 consecutive drops of $1 \%$ uranyl acetate (UAT) with tylose (1\% UAT in deionized water (diH2O), double filtered through a $0.22 \mu \mathrm{m}$ filter), blotted, then quickly aspirated to cover the sample with a thin layer of stain. Grids were imaged on a Hitachi 7600 TEM operating at $80 \mathrm{kV}$ with an AMT XR80 CCD (8 megapixel).

\section{Single Particle Interferometric Reflectance Imaging Sensing (SP-IRIS)}

EVs diluted $1: 100$ in DPBS were diluted $1: 1$ in incubation buffer (IB) and incubated at room temperature on ExoView R100 (NanoView Biosciences, Brighton, MA) chips printed with anti-human CD81 (JS-81), anti-human CD63 (H5C6), anti-human CD9 (HI9a) and antimouse-IgG1 (MOPC-21). After incubation for 16 hours, chips were washed with IB 4 times for 3 minutes each under gentle horizontal agitation at $500 \mathrm{rpm}$. Chips were then incubated for $1 \mathrm{~h}$ at RT with fluorescent antibodies anti-human CD81 (JS-81, CF555) and anti-human CD63 (H5C6, CF647) diluted 1:200 in a 1:1 mixture of IB and blocking buffer. Anti-human CD9 was not used, since the third wavelength was needed for the palmGRET reporter protein. The chips were subsequently washed once with IB, three times with wash buffer, and once with rinse buffer (all washes 3 minutes at $500 \mathrm{rpm}$ agitation). Chips were immersed twice in rinse buffer for 5 seconds and removed at a $45^{\circ}$ angle to remove the liquid from the chip. All reagents and antibodies were obtained from NanoView Biosciences (\#EV-TETRAC). All chips were imaged in the ExoView scanner (NanoView Biosciences) by interferometric reflectance imaging and fluorescent detection. Data were analyzed using ExoView Analyzer 3.0 software. Fluorescent cutoffs were as follows: CF555 channel 300 a.u., CF488 channel 410 a.u., CF647 channel 300 a.u., allowing $<1 \%$ of particles above background in the isotype control. Fluorescent counts from multiple measurements were normalized against the total fluorescent particle count. 


\section{Imaging flow cytometry}

EVs diluted $1: 10$ in DPBS were quantified by imaging flow cytometry on an Amnis ImagestreamX MkII instrument (Amnis Corp, Seattle, WA) on low flow speed, using a 60x objective and extended depth of field (EDF) option enabled. EGFP signal was collected in channel 2 (480-560 nm filter), MemGlow 700nm signal was collected in channel 5 (642-745 $\mathrm{nm}$ filter), and sideward scatter (SSC) was collected in channel 6 (745-800 nm filter). Negative controls recommended by the MiFlowCyt-EV consortium ${ }^{30}$ were included in all measurements: buffer only control, free dye control (200 nM MemGlow700 in PBS), single stained EVs (palmGRET only, and MemGlow700 labelled EV from untransfected Expi293F cells). Serial dilutions were included to ensure measurement in the linear range of the instrument, and to rule out swarm effects. Data were analyzed using Amnis IDEAS software v6.2.

\section{In vivo administration}

\section{Mice}

Balb/cJ mice (Jackson Labratories, 8-12 weeks, female) were injected intraperitoneally with $100 \mu$ fluorofurimazine (Su et al., Nat Methods 2020) under sedation with isoflurane. Subsequently 1.5E11 EVs were administered by tail-vein injection or intranasal instillation. Bioluminescent imaging was performed on a Caliper IVIS SpectrumCT In Vivo Imaging System (Caliper Life Sciences, Hopkinton, MA, USA). Images were taken every 30 seconds (exposure time $=30 \mathrm{~s}$ ) in luminescence mode. Mice were perfused with PBS via cardiac puncture 40 minutes after EV administration, organs were harvested and imaged exvivo in fluorescence mode (ex 689, em 713). Tissues were homogenized in $1 \mathrm{ml} \mathrm{N}$-PER (brain) or T-PER (other organs) + Complete Mini protease inhibitor cocktail tablet (Roche, Mannheim, Germany) in FastPrep Lysing Matrix D tubes on a FastPrep homogenizer. Homogenates were centrifuged for $5 \mathrm{~min}$ at $10,000 \mathrm{~g}$ at $4^{\circ} \mathrm{C}$, supernatant was taken off and used in NanoGlo and BCA assays (Pierce, Rockford, IL). Mice experiments were performed under approval of the Johns Hopkins University Animal Care and Use Committee (ACUC), study number M018M145.

\section{Macaques}

Juvenile pigtailed macaques (Macaca nemestrina, male, 3-4 years old) were obtained from the JHU pigtail colony. EVs (7E10 EVs, with 5-fold increments for all subsequent doses) were administered by intravenous injection into the small saphenous vein or by intranasal instillation through a catheter under ketamine sedation (10 mg/kg body weight). Macaques remained sedated during blood/CSF collection for the first hour by administering ketamine in $10-20 \mathrm{mg}$ increments, and were sedated again with $10 \mathrm{mg} / \mathrm{kg}$ body weight at the $4 \mathrm{~h}$ and $24 \mathrm{~h}$ timepoints. After each EV dose and biofluid collection, macaques were given two weeks to recover until the next EV administration, for a total of 5 doses. To assess the turnover rate of EVs in CSF, we administered 3E10 EVs via intrathecal injection, and collected blood and CSF after $0,0.5,1,3,6$, and 24 hours. Macaque experiments were performed under approval of the Johns Hopkins University Animal Care and Use Committee (IACUC).

At each timepoint, $500 \mu \mathrm{l}$ blood was collected by venipuncture into tubes containing $100 \mu \mathrm{l}$ ACD. Blood was processed within $1.5 \mathrm{~h}$ after collection by centrifugation for 5 minutes at 800 $\mathrm{g}$, plasma was taken off and stored directly at $-80^{\circ} \mathrm{C}$. To collect PBMC from the same sample, the blood cell pellet was reconstituted to $1 \mathrm{ml}$ with DPBS, carefully layered onto a 1 $\mathrm{ml}$ Histopaque-1077 cushion (Sigma, St. Louis, MO), and centrifuged for $30 \mathrm{~min}$ at $400 \mathrm{~g}$ without brake. After discarding the supernatant layer, the PBMC-containing interphase was 
transferred to a new tube. PBMCs were washed twice by adding $1 \mathrm{ml} \mathrm{PBS}$, centrifuging for 10 min at $250 \mathrm{~g}$, and discarding the supernatant. The final PBMC pellet was taken up in 200 $\mu \mathrm{l}$ lysis buffer (PBS $+1 \%$ Triton-X100 + Complete Mini protease inhibitor cocktail tablet (Roche, Mannheim, Germany)), lysed on ice for 15 minutes. Nuclei were removed by centrifuging 15 minutes at $16,000 \mathrm{~g}$ at $4^{\circ} \mathrm{C}$. Protein concentration was determined by BCA assay (Pierce, Rockford, IL). Per timepoint, $500 \mu \mathrm{l} \mathrm{CSF} \mathrm{was} \mathrm{collected} \mathrm{which} \mathrm{was} \mathrm{centrifuged}$ for 10 minutes at $2000 \mathrm{~g}$ to remove cells. The supernatant was taken off and stored directly at $-80^{\circ} \mathrm{C}$. After the final dose, macaques were euthanized using Nembutal $(20-30 \mathrm{mg} / \mathrm{kg})$ and perfused with PBS. Organs were excised and snap-frozen at $-80^{\circ} \mathrm{C}$. Parts of the spleen and bronchial lymph nodes were processed directly for flow cytometry (see below for details).

\section{Flow cytometry}

PBMCs were immunolabeled directly in whole blood with fluorescent antibodies. $100 \mu \mathrm{l}$ whole blood was added to antibody cocktails (mo-anti-CD159a-PE, Beckman Coulter, cat\# IM3291U, dil 1:30; mo-anti-CD4-PerCP/Cy5.5 BD Biosciences 552838 dil 1:7.5; mo-antiCD20-e450 Thermo Fisher, cat\# 48-0209-42 dil 1:60; mo-anti-CD3-V500 BD, cat\# 560770, dil 1:30; mo-anti-CD8-BV570, BioLegend, cat\# 301038, dil 1:60; mo-anti-CD14-BV650, BioLegend, 563419, dil 1:30), briefly vortexed and incubated at room temperature for 20 minutes. Next, red blood cells were lysed by addition of $2 \mathrm{ml} \mathrm{RBC}$ lysis buffer (ACK lysing buffer $0.83 \% \mathrm{NH}_{4} \mathrm{Cl}, 0.1 \% \mathrm{KHCO}_{3}, 0.03 \%$ EDTA) and incubation at room temperature for 10 minutes. Tubes were centrifuged at $400 \mathrm{~g}$ for 5 minutes and supernatant was discarded. Next, $2 \mathrm{ml}$ PBS was added and tubes were centrifuged again at $400 \mathrm{~g}$ for 5 minutes. Supernatant was discarded, labelled PBMCs were carefully resuspended in $500 \mu \mathrm{PBS}$ and measured directly on a BD LSR Fortessa flow cytometer. As negative controls, we included whole blood collected before the injection of EVs, and fluorescence minus one (FMO) controls for CD159a and CD4 to allow for accurate gating of GFP, PE, and PerCP/Cy5.5 fluorescence. Cells from the spleen and bronchial lymph nodes were mechanically isolated from freshly excised tissues using 18-gauge needles in cold RPMI and passed through a $100-\mu \mathrm{m}$ cell strainer. Spleen cells were lysed using RBC lysis buffer. $10^{6}$ cells were resuspended in $100 \mu$ l of PBS $2 \%$ FBS solution for antibody staining using the same antibody cocktail as the PBMCs.

\section{Nanoluciferase assays}

Purified EV samples and SEC fractions were diluted 20-fold in PBS and were loaded in a white plastic 96 well plate, $50 \mu \mathrm{l}$ per well in duplicates. Biofluid samples, PBMC lysates and tissue homogenates, were loaded undiluted at $50 \mu \mathrm{l}$ per well in duplicates. Nano-Glo substrate (furimazine, Promega, Madison, WI) was diluted $1: 50$ in assay buffer according to the manufacturer's instructions. $50 \mu$ diluted Nano-Glo reagent was added per well, and bioluminescence was measured immediately on a Fluoroskan Ascent plate reader (software v6.2) in bioluminescence mode, integration time $20 \mathrm{~ms}$.

\section{Statistics and EV half-life calculation}

Statistical differences were determined by one-way ANOVA with Tukey's post-hoc test in GraphPad Prism 9.1, differences with $p<0.05$ were considered to be statistically significant. EV half-life in biofluids was determined by linear regression of the nanoluciferase signal versus time on a log-lin chart. EV half-life was calculated from the slope of the regression line: $\mathrm{t}_{1 / 2}=\log (2) /$ slope

\section{Availability of protocols}

Written details on experimental procedures have been submitted to the EV-TRACK 
bioRxiv preprint doi: https://doi.org/10.1101/2021.07.28.454192; this version posted July 29, 2021. The copyright holder for this preprint (which was not certified by peer review) is the author/funder, who has granted bioRxiv a license to display the preprint in perpetuity. It is made available under aCC-BY 4.0 International license.

knowledgebase ${ }^{31}$. High resolution flow cytometry experiments were performed following the MIFlowCyt-EV guidelines ${ }^{30}$. 


\section{Results}

Production, separation, and general characterization of labelled EVs. To study the pharmacokinetics and biodistribution of EVs in larger animals such as macaques, highly sensitive reporters are required that can be detected with a high signal-to-background ratio. Therefore, we used two state-of-the art EV reporters: the near-infrared, self-quenching membrane dye MemGlow $700^{28}$, which was previously used to track tumor EV in live zebrafish ${ }^{29}$, and the dual reporter protein palmGRET (palmitoylated EGFP-Nanoluciferase), which was previously used to track tumor EVs in mice ${ }^{22}$. We transiently expressed palmGRET in Expi293F suspension cells (Supplementary Figure 1A) and harvested the conditioned culture medium three days later. EVs were concentrated by tangential flow filtration, labelled with MemGlow 700, concentrated further by ultrafiltration, and subjected to size exclusion chromatography (SEC) to separate EVs from free dye and non-EV-associated proteins (Supplementary Figure 1B). To satisfy the MISEV criteria ${ }^{32}$, we extensively characterized individual SEC fractions and pools by microBCA, SDS-PAGE and Western blot. A small protein peak was observed in SEC fractions 1-4 (Supplementary Figure 1C and D) which was positive for EV markers CD63, CD9, and TSG101 but devoid of ER marker calnexin (Figure 1A), indicating that EVs were isolated with minimal contamination by other cellular material. Transmission electron microscopy (TEM) and nanoparticle tracking analysis (NTA) of pooled fractions 1-4 confirmed the presence of EVs with expected morphology and an average diameter (NTA) of $122.6 \mathrm{~nm}$ (SD +/- $9.9 \mathrm{~nm}$ ) (Figure 1B and C).

Characterization of label incorporation. We used nanoluciferase assay to determine the presence of palmGRET in different SEC fractions (Figure 1D). Nanoluciferase was present in EVs (fractions 1-4) but was also highly abundant as free protein in the later protein fractions $(7-11)$, highlighting the importance of size-based separation of EVs from non-EV proteins. An overview of the characteristics of different EV batches can be found in Supplementary Table 1. To confirm the incorporation of MemGlow 700, we used the Amnis Imagestream ISX imaging flow cytometer, which allows near-infrared detection and is suited to characterize small EVs ${ }^{33,34}$ (Supplemental Figure 2). To set gates for double-positive EVs, we used control EVs that contained only palmGRET or only MemGlow 700

(Supplemental Figure 2A). We performed serial dilutions to rule out coincidence events (Supplemental Figure 2B). Detergent treatment resulted in strongly reduced event counts, confirming that the measured events were indeed membrane particles (Supplemental Figure 2C) in accordance with the MIFlowCyt-EV recommendations ${ }^{30}$. On average, $30 \%$ of the EVs were double-positive for both reporters (Supplemental Figure 2D), while hardly any double-positive events were detected in unlabeled EVs, PBS, or free dye controls. Next, we used SP-IRIS to investigate the colocalization of palmGRET with the major EV tetraspanins CD9, CD63, and CD81 (Figure 1E). We observed the most palmGRET signal in EVs captured by anti-CD63 antibodies, and slightly less in EVs displaying CD81 and CD9, indicating that each of these tetraspanins was present in the palmGRET-labelled EV population. Furthermore, we observed considerable colocalization of tetraspanins. Next, we performed a detergent/protease protection assays to confirm that palmGRET is enclosed within a lipid bilayer (Figure 1F). Protease K treatment of palmGRET EVs alone did not affect the nanoluciferase signal, whereas addition of detergent lead to a reduction in signal. This confirmed that palmGRET was enclosed within the lumen of EVs, as previously reported ${ }^{22}$.

Stability and detection in blood plasma. Prior to in vivo studies, we sought to determine detectability of labelled EVs in the biological matrix of blood plasma. This was done not only to assess assay sensitivity, but also because factors in blood might reduce stability of signal 
or contribute to background. palmGRET EVs were spiked into plasma, and serial dilutions were prepared over a 100,000-fold range (Figure 1G). Nluc signal was detected above background levels (macaque plasma without EVs) over the entire dilution range. We compared the Nluc signal with the number of particles per well (calculated from NTA particle counts and dilution factor), which suggested that the limit of detection was approximately around $200 \mathrm{EVs} / \mu \mathrm{l}$ (detection limit: 10,000 EVs in $50 \mu \mathrm{l}=200 \mathrm{EV} / \mu \mathrm{l}$ ). These findings supported further use of the model to evaluate palmGRET EV pharmacokinetics and biodistribution.

Design and dosing: in vivo study. We next compared intravenous and intranasal administration of different amounts of EVs, tracking the abundance of EVs in plasma and CSF over 24 hours after each administration (Figure 2A). The starting dose was based on a previous study in which EVs from 4E7 mesenchymal stem cells were administered into sheep fetuses ${ }^{25}$. We used a comparable number of cells to produce EVs for our starting dose. Initial measurements showed that Expi293F cells produced about 1.7E3 EV/seeded cell under the culture conditions we used. This set the starting dose to approximately 7E10 EVs (4E7 cells $x 1.7 \mathrm{E} 3 \mathrm{EV} /$ cell $\approx 7 \mathrm{E} 10 \mathrm{EVs}$ ). Three subsequent EV doses were administered at 5 -fold greater concentration each time, with several weeks between doses. The fourth and highest dose was then administered a second time.

Detection of EVs in blood plasma. Intranasal administration of EVs resulted in little if any nanoluciferase detection in plasma at any timepoint and after any dose (Figure 2B). This suggested that Expi293F EVs might have remained in the nasal cavity, mucosa, or lungs after administration, and did not enter the bloodstream. In contrast, intravenously administered EVs could be reliably detected in plasma at all doses (Figure 2C). For the three lowest doses (7E10, 4E11 and 2E12 EVs), nanoluciferase signal could be detected in plasma up to $24 \mathrm{~h}$ (1440 minutes) after administration. Unexpectedly, the fourth and highest dose (9E12 EVs, magenta data points) was cleared more rapidly from plasma than dose 3 , and was hardly detectable above background after 4 hours (360 minutes). We doublechecked the particle concentration and nanoluciferase signal in this particular EV dose, to rule out any issues with storage or handling, but we did not observe any abnormalities that could explain the observed lower signal and increased clearance. When we repeated administration of this highest dose (black data points), we observed a comparable clearance pattern.

Half-life of EVs in plasma. Next, we used these data to calculate the half-life of EVs in plasma. The data followed a biphasic decay profile on a log-lin chart, with rapid decay shortly after administration followed by slower decay at a later timepoint, described by a twocompartment pharmacokinetic model ${ }^{35}$. We calculated the half-life from the data points during the first 60 minutes (Figure 2D), which ranged between 36 to 42 minutes for the three lowest doses. In contrast, the half-life of signal after administration of the highest dose was approximately 11 minutes. Because we traced the clearance of the repeat of the highest dose for 15 minutes, the half-life of repeat dose 4 could not be reliably determined.

Detection of EVs in CSF. After intranasal administration (Figure 2E), EVs could not be reliably detected above background in CSF at any timepoint and after any dose. In contrast, signal was observed in CSF after intravenous administration at the higher doses (Figure 2F). EV signal in CSF peaked after 30 minutes for dose 4 and at 60 minutes for dose 3 , and remained detectable above background for up to 24 hours. Intravenous doses 1 and 2 did not lead to detectable CSF at any of the timepoints. Intravenously administered EVs may thus migrate from plasma into CSF, at least at higher doses. 
Half-life of EVs in CSF and detection in plasma after intrathecal injection. We next questioned whether EV half-life in CSF is comparable to that in plasma. To achieve initial levels of EVs in CSF that were similar to those administered into blood, we introduced 3.2E10 EVs directly into the CSF of a previously untreated subject via intrathecal injection, followed by collection of CSF and plasma at regular intervals (Supplementary Figure 3). Strong nanoluciferase signal in CSF remained detectable up to 6 hours. Based on data from the first hour post-treatment, EV half-life in CSF was approximately 12.5 minutes, considerably shorter than half-life in plasma. Meanwhile, nanoluciferase signal was detected above background in plasma only at later timepoints, suggesting that EVs might be able to diffuse from CSF into plasma, albeit at relatively low levels.

Uptake of EVs by PBMCs. Since circulating white blood cells contribute to clearance of EVs from blood ${ }^{36}$, we investigated uptake of EV-associated signal by peripheral blood mononuclear cells (PBMCs) at different timepoints following EV administration. Specifically, we isolated PBMCs from the blood samples collected in the $24 \mathrm{~h}$ after administration, lysed them, and measured the amount of nanoluciferase taken up by these cells. After intranasal administration (Figure 2G), no EV uptake could be detected in the PBMCs at any of the doses, consistent with the absence of EV in blood plasma. In contrast, intravenous administration at all doses led to EV uptake as soon as 1 minute after injection (Figure $\mathbf{2 H}$ ). At the highest doses, nanoluciferase was detectable in PBMCs up to one hour. There was also a linear relationship between total nanoluciferase signal (all time points combined) and dose for the highest three doses (Figure 2I). The repeat dose 4 was not included since blood samples were only collected during 15 minutes.

PBMC subtypes responsible for EV uptake. We next sought to determine the PBMC subtype(s) responsible for rapid uptake of EVs in blood. Flow cytometry was performed with PBMCs from whole blood samples collected during the first 10 minutes after administration of the highest EV dose (Figure 3A), using an antibody panel that identifies several PBMC subtypes: monocytes (CD159- CD3- CD20- and CD14+ or CD14-), T cells (CD3+ and CD4+ or CD8+), B cells (CD3- CD20+), and NK cells (CD159+). EVs were detected through their internal GFP label as well as by presence of the MemGlow self-quenching lipid dye. The gating strategy is depicted in Supplementary Figure 4A. We observed EV uptake in monocytes, $C D 3+$ lymphocytes and $C D 20+B$ cells already at 1 minute after administration (Figure 3A), consistent with nanoluciferase results from PBMC lysates (Figure 2H). Monocytes, CD3+ cells, and B cells differed in EV uptake efficiency; $80.8 \%$ of B cells became GFP+, while $13.8 \%$ of monocytes and $6.7 \%$ of CD3+ cells became GFP+. Of the CD3+ cells, both CD4+ and CD8+ T cells became GFP-positive to a similar extent (Supplementary Figure 4B). NK-cells did not efficiently take up EVs (Supplementary Figure 4C). We found similar percentages of EV uptake after repeating administration of the highest EV dose (Figure 3B). Gating of GFP+ or MemGlow+ cells largely showed similar uptake kinetics for both EV markers. However, B cells efficiently took up both GFPcontaining and MemGlow-containing EV, while CD3+ cells seemed to prefer GFP-containing EVs. Since monocytes were less abundant than B cells and T cells, the percentage of MemGlow-positive monocytes could not be reliably determined. Taking into account both uptake efficiency and cell type contribution to the overall PBMC population, B cells made the largest contribution to EV uptake ( $7 \%$ of all GFP+ mononuclear cells were B cells), followed by $\mathrm{T}$ cells $(\sim 4 \%)$, while monocytes took up less than $1 \%$. In total, $11 \%$ of the mononuclear cells took up GFP-containing EVs (Figure 3C).

Organ biodistribution in mouse. We also investigated how intranasal and intravenous administration affected distribution of our labelled EVs to different organs. For this part of the study, we performed initial experiments in mice, since their small size makes them suitable 
for in vivo and ex vivo imaging. For in vivo imaging, we used a modified nanoluciferase substrate that is more water-soluble than regular Nano-Glo, fluorofurimazine (FFz), allowing better distribution of the substrate throughout the whole animal ${ }^{37}$. After interperitoneal injection of FFz, we administered 1.4E11 EVs by intravenous or intranasal routes and measured bioluminescence (Figure 4A). After intranasal administration, we observed bright signal in the nasal cavity and in some cases also in the lungs. After intravenous administration, we observed most signal in the liver. After 40 minutes, we perfused the mice, harvested the organs and measured near-infrared fluorescence ex vivo (Figure 4B). Most signal was observed in lungs (intranasal) or liver (intravenous), in line with our in vivo imaging observations. Next, we prepared tissue homogenates from all harvested organs and measured EV uptake by nanoluciferase assay (Figure 4C). Intranasal administration did not result in strong nanoluciferase signal in most organs, although we observed high but variable nanoluciferase signal in lung, in line with our in vivo imaging results. After intravenous administration, we observed strong nanoluciferase signal in the liver and spleen, consistent with earlier reports ${ }^{18,19,21-23}$. We additionally observed some EV uptake in kidney and lung, and to a lesser extent in heart, colon and brain. EV uptake in brain was lower for intranasal administration than for intravenous administration.

Organ biodistribution in macaque. EV biodistribution was next assessed by nanoluciferase assay of macaque tissues harvested 60 minutes after administration of the last, highest dose (9E12 EVs; Figure 5A). After intravenous administration, we observed strong signal in liver and spleen, in line with the rodent results. Some uptake in lung was observed after intravenous, but not intranasal administration. EV uptake by kidney, heart, colon and brain was limited for both administration routes. A faint nanoluciferase signal was observed in the medulla in brain after intravenous administration, suggesting this region might be the most accessible to EVs from the bloodstream. Nevertheless, the signal in medulla was low compared with signal in organs such as liver and spleen. Next, we used flow cytometry to identify which cell types in the spleen took up EVs (Figure 5B). After intravenous administration, we observed GFP+ monocytes and CD3+ lymphocytes, but the most efficient EV uptake was observed in $B$ cells, consistent with the PBMC results reported above. Interestingly, after intranasal EV administration, we also observed a small percentage of GFP+ CD3+ lymphocytes in the spleen (Figure 5C). 


\section{Discussion}

Half-life of EVs in plasma. To date, most preclinical studies on EVs have used mice, rats and zebrafish ${ }^{3,27}$, and, remarkably, we measured the circulation time of EVs in NHP $\left(t_{1 / 2}\right.$ approx. $40 \mathrm{~min}$ ) to be much longer than that reported in mice ( $\mathrm{t}_{1 / 2}$ approx. 5 minutes $)^{17,19,21-23}$ and zebrafish ${ }^{36}$. EVs remained detectable in plasma up to 24 hours. Possibly, species differences between EV producer cell and the recipient animal model may affect the stability of EVs. For example, it has been shown that murine lentiviral vectors were less stable in human serum compared with human lentiviral vectors ${ }^{38}$. Moreover, the stability of human lentiviral vectors in serum from different ape species decreased with increasing evolutionary distance ${ }^{39}$, possibly via complement- or antibody-mediated effects ${ }^{38,40}$. How the species of EV donor cells affects the stability of EVs in different animal species has not yet been addressed. A study that used MRI to compare the circulation time of oleic acid-coated iron nanoparticles between macaques and mice reported circulation times of $\sim 2 \mathrm{~h}$ for both animal species ${ }^{41}$. Since iron nanoparticles are not as degradation-sensitive as EVs and lack surface proteins that could facilitate cellular uptake, these longer circulation times are perhaps expected. Interestingly, the half-life of our EVs was in the range of that of HIV-1 virions infused into naïve macaques ${ }^{42}$. This study reported a half-life of 13.0-19.3 minutes based on viral RNA, and 22-29 minutes based on pelletable Gag p24 in plasma ${ }^{42}$. Considering that HIV-1 actively fuses with and infects target cells, it is not surprising that EVs, which are thought to lack a consistent fusion mechanism like those of enveloped viruses, may have slightly longer circulation times.

Repeated administration of EVs and immune response. An important consideration in potential EV therapeutics is whether repeated administration will provoke immune responses. If so, EVs might need to be prepared from autologous or allogeneic cells, as opposed to the much easier and cheaper alternatives of nonautologous or even xenogeneic materials ${ }^{43,44}$. In our study, EVs from a human cell source were cleared markedly more rapidly after the $4^{\text {th }}$ and $5^{\text {th }}$ administrations (repeated administration of the highest dose) than after administrations at lower doses and weeks earlier. Although it is formally possible that EV clearance rate is somehow linked to initial concentration, the observation is also consistent with potential development of immune responses specific to EVs. Supporting the immune response hypothesis, a previous study showed that the half-life of HIV-1 was markedly decreased (3.9-7.2 minutes) in macaques with neutralizing antibodies against HIV1 gp120, compared with naïve macaques (13.0-19.3 minutes) ${ }^{42}$. Similarly, although PEGylation is commonly used to increase half-life of nanoparticles including liposomes and $E V s^{19}$, repeated administration leads to accelerated blood clearance ${ }^{45}$ mediated by PEGspecific IgM ${ }^{46}$. Notably, EV-based vaccines from several sources can also elicit strong immune responses ${ }^{47-53}$. Therefore, although some results have indicated limited immunogenicity of specific EVs in specific models, immune responses to EVs require additional investigation.

Uptake by PBMC subtypes. Our results differ somewhat from previous findings in that, although multiple PBMC subtypes took up EV label, B cells appeared to take up EVs more efficiently than several other PBMC types. Once thought to be non-phagocytic, B cells are now known to have phagocytic capacity ${ }^{54}$. Indeed, a previous study also showed that human plasma EVs can be taken up by $B$ cells ${ }^{55}$. However, in that study, the majority of uptake was observed in monocytes ${ }^{55}$. Previous in vivo work, e.g., with clodronate-depleted monocyte/macrophage populations ${ }^{56}$ and with blockade of scavenger receptor class $A^{57}$, also implicated monocytes/macrophages in EV clearance. Possibly, the biological source of EVs, reporter system, species differences, and age of the subject contribute to these apparent differences. The rapid depletion of EV-associated signal from these cells is also 
worth noting, and could be due to degradation of EVs or to clearance of cells. Interestingly, within one hour of IV administration, we detected GFP+ B cells and monocytes in the spleen, suggesting rapid trafficking of blood $B$ cells and monocytes to the spleen (and possible elsewhere) after EV uptake.

Uptake in CSF, but not in brain. A striking result of our study was the low level of EV uptake into central nervous system compartments, regardless of administration route. First, intravenous administration: acceptance of the ability of EVs to cross the blood-brain barrier in both directions has become so widespread that statements to this effect are often not even referenced in the literature. Yet much of the evidence for EV transfer across the BBB is indirect. After intravenous administration, EVs might enter brain tissue directly across the BBB. They might also traverse the choroid plexus epithelium, a more permeable counterpart to the blood-brain barrier ${ }^{58}$. We detected low levels of EVs in brain tissue (especially medulla) and in CSF following IV administration, albeit at much lower levels than in peripheral compartments. In CSF, signal remained detectable at 24 hours. This result is similar to findings of a study of IV insulin in dogs, in which rapid clearance from plasma was followed by detection in CSF, peaking around 90 minutes ${ }^{59}$. However, we are not convinced that entry into CSF is an efficient precursor to brain entry. We did not observe substantial EV uptake into brain even after injecting EVs directly into the CSF (Driedonks and Witwer, data not shown). This suggests that reaching the CSF may not be enough to gain entry to the brain. Overall, the low levels of signal in both tissue and CSF suggest that the EV bloodbrain route in our model is more of a precarious footpath than a superhighway.

Negligible brain uptake after IN administration. Our results suggest that IN delivery of EVs to the brain in large animals should not be a foregone conclusion. Certainly, numerous studies report that IN EVs or their presumed cargo enter the brain parenchyma (see, for example $\left.{ }^{60-65}\right)$. As a result, intranasal delivery of EVs is thought to be a promising way to treat CNS disease. However, the studies with positive results that we are aware of have all been performed in mouse or rat models, and predominantly with EVs sourced from MSCs or other stem cells. Physiology of the recipient species or characteristics of the source cells could explain disparate results. Previously, studies of intranasally administered recombinant vesicular stomatitis viral vectors ${ }^{66}$ and a nanogel pneumococcal vaccine formulation ${ }^{67}$ in macaques found no brain uptake. In any case, our findings suggest that intranasal delivery of EVs must now be assessed in multiple models to determine if this route is truly an EV delivery option for large animals including humans.

Intranasal delivery factors could also influence outcome. Nebulization or aerosolization could be tested in large animals, in addition to instillation, and repeated short-term administrations might also be useful. Each result should also be carefully interpreted in light of the separation and characterization of the administered EVs: how sure are we that signal was associated with EVs before administration? For example, a study finding efficient intranasal administration used MSC EVs that were incubated with gold nanoparticles and then ultracentrifuged for two hours at $100,000 \mathrm{~g}^{20}$. Although these procedures were meant to label EVs and then remove free GNPs, it is unclear how efficient the labelling was and also doubtful that ultracentrifugation would separate EV-associated GNPs from free particles. Free GNPs might thus have contributed to or fully explained these results.

EV uptake by peripheral organs. In both macaques and mice, IV-administered EVs were most efficiently taken up by the liver and spleen, followed by lung. This is in accordance with many other EV biodistribution studies in mice ${ }^{18,19,21-23}$. In mice, we also observed low levels of uptake by kidney and, to a lesser extent, heart and colon. Thus, peripheral biodistribution of IV EVs was largely comparable between macaques and mice, with the exception of 
differences in kidney and lung. In mice lungs, large EVs may be retained in the narrow microcapillaries $(1-2 \mu \mathrm{m})^{27}$. Wider capillaries of macaques might explain the relatively reduced retention of EVs. Intranasal administration of EVs in mice resulted in variable uptake in lungs, with negligible uptake in other organs. In vivo imaging revealed that the nasal cavities were the main retention site for EVs. Nasal epithelia are covered by a mucosal layer that acts as a barrier against bacteria and viruses. Possibly, this mucus layer may prevent EVs from migrating into the lungs. Previously, it was shown that infusion of larger volumes leads to more entry into the lungs, instead of just the nasal cavity ${ }^{68}$.

Factors beyond administration route that affect EV biodistribution may include differences in tetraspanins or other surface proteins, EV labeling strategy ${ }^{21,23}$, and the type of EV donor cells ${ }^{18,69}$. Illustrating this, endogenous CD63-Nluc EVs from cardiomyocytes were shown to target different organs than CD63-Nluc EVs from Expi293F cells ${ }^{23,69}$. Additionally, the disease status of the recipient may affect tissue accumulation. For example, the uptake of MSC-EVs in kidneys was increased in mice with acute kidney injury compared to healthy controls ${ }^{70,71}$. Future research will likely highlight other factors that control $\mathrm{EV}$ tissue retention (or even true "homing" or "targeting," if this is possible for EVs ${ }^{13}$ ), and will give clues on how to better control the fate of EVs in vivo.

Taken together, nanoluciferase-based reporters allowed sensitive tracking of EVs in larger animals for pharmacokinetic measurements. We show that EVs from human cells had a longer circulation time in macaques compared with mice, but that CNS penetration was low for both IV and IN administration. We found that repeated administration leads to more rapid clearance, which may have implications for EV-based therapies against cancer and immune diseases. We hope that our findings from this baseline study in macaques will help to inform future research and therapeutic development of EVs.

\section{Conflict of interest}

The authors report no conflicts of interest.

\section{Funding statement}

This study was supported by the US National Institutes of Health through Al144997 (to KWW) and U42OD013117 (to EKH). The Witwer lab is also supported in part by the NIH through DA047807, MH118164, and CA241694, and by the Michael J. Fox Foundation (Grant 00900821). CPL was supported by the Ministry of Science and Technology (MOST) grants (109-2628-B-001-032) and Academia Sinica Career Development Award (AS-CDA109-M04).

\section{Author contributions}

Tom Driedonks, Kelly Pate, and Kenneth Witwer conceived the research and designed experiments. Tom Driedonks, Linglei Jiang, Zheng Han, Guanshu Liu, Erin Shirk, Olesia Gololobova, Lyle Nyberg, Gabriela Lima and Barbara Smith performed experiments. Bess Carlson, Suzanne Queen, Zheng Han, Guanshu Liu, Kayla Schonvisky, Natalie Castell, Mitchell Stover, Selena Guerrero-Martin, Riley Richardson, Jessica Izzi, Eric Hutchinson and Kelly Pate performed animal experiments. Charles Lai designed and provided the palmGRET reporter construct. Tom Driedonks and Kenneth Witwer wrote the manuscript. All authors reviewed and revised the manuscript. 
bioRxiv preprint doi: https://doi.org/10.1101/2021.07.28.454192; this version posted July 29, 2021. The copyright holder for this preprint

(which was not certified by peer review) is the author/funder, who has granted bioRxiv a license to display the preprint in perpetuity. It is made available under aCC-BY 4.0 International license.

\section{Acknowledgements}

The authors thank Weiying Zhang and Tricia Nilles for assistance with the imaging flow cytometry measurements. 


\section{Figure legends}

Figure 1 - Characterization of palmGRET EVs A) EVs produced by Expi293F cells were concentrated by TFF and ultrafiltration, followed by size-exclusion chromatography (SEC). Equal volumes of individual SEC fractions, pooled EV (fr 1-4) and cell lysate were analyzed by SDS-PAGE and Western blot for EV markers CD63, CD9, Tsg101 and ER marker (i.e., cytosolic contaminant marker) Calnexin. Blot is representative of $n=6$ repeats. B) Pooled EV and protein fraction 10 (RNP) were imaged by negative stain transmission electron microscopy. Scale bars represent $100 \mathrm{~nm}$. Image is representative for $\mathrm{n}=6 \mathrm{EV}$ batches. $\mathrm{C}$ ) Particle size and concentration of pooled EV were determined by NTA. Average of $n=5 \mathrm{EV}$ batches is shown. D) SEC fractions $1-11$ were diluted 20x and the presence of the palmGRET reporter was validated by Nano-Glo assay. Measurement of $n=5 \mathrm{EV}$ batches are shown. E) SP-IRIS was used to determine the co-localization of palmGRET with the major tetraspanins. Total counts is the number of total fluorescent spots of $n=4 \mathrm{EV}$ batches that are captured by CD63, CD81, CD9 and isotype antibodies. CD81 (green) and CD63 (red) graphs show co-localization of these markers with capture tetraspanins as \% of total counts. PalmGRET shows the colocalization of the GFP signal from the palmGRET reporter with capture tetraspanins as $\%$ of total counts. Average of $n=4$ EV batches, ${ }^{*} p<0.05,{ }^{* *} p<$ 0.01 , one-way ANOVA with Tukey's post-hoc test. F) Pooled EV were incubated with protease $\mathrm{K}$ and Triton, protease $\mathrm{K}$ alone, or without additives. Resistance of the palmGRET reporter to different treatments was determined by Nano-Glo assay. Average of $n=3$ batches, ${ }^{* *} p<0.01$, one-way ANOVA with Tukey's post-hoc test. G) Pooled EV were spiked into plasma of a healthy macaque, diluted in twofold serial dilutions from 160x $-327680 x$, and measured by Nano-Glo assay. Nano-Glo data were plotted against the dilution factor (left) and theoretical EV concentration from NTA (right)

\section{Figure 2 - Pharmacokinetics of palmGRET EV administered intravenously versus intranasally in macaques $A$ ) Schematic of the study setup. EVs were administered} intravenously (IV) or intranasally (IN) into macaques, over a 125-fold dose range. Blood and CSF were sampled before administration, and were collected at timepoints indicated in the chart. B) Detection of palmGRET EVs in plasma at different timepoints after intranasal administration by Nano-Glo assay. C) Detection of palmGRET EVs in plasma at different timepoints after intravenous administration by Nano-Glo assay. D) EV half-life versus EV dose, from the data in Figure $2 \mathrm{C}$. Nluc was plotted on a log-axis and $t$ on a linear axis, the half-life was calculated from the slope between $t=2$ and $t=60$, using the formula $t 1 / 2=$ $\log (2) /$ slope. E) Detection of palmGRET EVs in CSF at different timepoints after intranasal administration by Nano-Glo assay. F) Detection of palmGRET EVs in CSF at different timepoints after intravenous administration by Nano-Glo assay. G) Detection of palmGRET EVs in PBMC lysates at different timepoints after intranasal administration by Nano-Glo assay. $H$ ) Detection of palmGRET EVs in PBMC lysates at different timepoints after intravenous administration by Nano-Glo assay. I) Total nanoluciferase signal detected in PBMC lysates during the first 60 minutes after administration was calculated from Figure $2 \mathrm{H}$, and plotted against the EV dose. Throughout, error bars represent the standard error of the assay.

Figure 3 - EV uptake by PBMC subsets quantified by flow cytometry A) Whole blood collected after intravenous administration of the highest dose (dose 4) was immunolabeled, after which uptake of palmGRET (GFP)-containing EVs was measured by flow cytometry. Full gating strategy is found in Supplementary Figure 4A. EV uptake in monocytes (top), 
CD3+ lymphocytes (middle) and CD20+ B cells (bottom) was determined as \%GFP+ cells as percentage of the total $\mathrm{B}$ cells, CD3+ lymphocytes, or monocytes, respectively. Plots are representative for $n=2 \mathrm{EV}$ administrations into the same animal, two weeks apart. $B$ ) Quantification of \%GFP and \% MemGlow-positive PBMC subsets from Figure 3A, as percentage of the total $B$ cells, CD3+ lymphocytes, or monocytes, respectively. Data from $\mathrm{n}=2 \mathrm{EV}$ administrations are shown. C) Quantification of GFP positive mononuclear cells (MNC), CD3+ lymphocytes, CD20+ B cells, and monocytes, relative to the total pool of MNC. Data from $\mathrm{n}=2 \mathrm{EV}$ administrations are shown.

\section{Figure 4 - Biodistribution of palmGRET EVs administered intravenously or} intranasally into mice A) Female Balb/cJ mice were injected intraperitoneally with NanoGlo substrate (fluorofurimazine, FFz), after which 1.4E11 EVs were administered intranasally (IN) or intravenously. In vivo biodistribution was monitored on a bioluminescent imager. Two intranasal administrations are shown (different outcomes), as well as one intravenous administration and one control, representative of $n=2$ experiments. B) Ex-vivo imaging of mouse organs harvested 40 minutes after EV administration, in fluorescence mode (ex 698, em 713). Images are representative for $n=2$ experiments. C) $E V$ uptake in mouse tissues was determined by Nano-Glo assay on tissue homogenates. Data from $n=5$ animals are shown. Statistical differences were determined by one-way ANOVA with Tukey's post-hoc test, ${ }^{*} p<0.05,{ }^{* *} p<0.01$, and ${ }^{* * *} p<0.001$.

\section{Figure 5 - Biodistribution of palmGRET EVs administered intravenously or} intranasally into macaques A) Organs were collected 60 minutes after IV or IN administration of the highest dose of EV (dose 4 repeat). EV uptake in macaque tissues was determined by Nano-Glo assay on tissue homogenates. Data from 1 animal per group are shown; dots indicate replicate measurements of the same sample. B) Spleen was homogenized and immunostained directly for flow cytometric analysis of EV uptake by PBMCs in the spleen after intravenous administration. Gating strategy was the same as for whole blood PBMCs, see Supplementary Figure 4A. Dot plots show the \%GFP+ cells as percentage of the total $B$ cells, CD3+ lymphocytes, or monocytes, respectively. $N=1$ measurement. C) Similar as in B, EV uptake by PBMCs in the spleen after intranasal administration. Dot plots show the \%GFP+ cells as percentage of the total B cells, CD3+ lymphocytes, or monocytes, respectively. $\mathrm{N}=1$ measurement.

\section{Supplemental Figure 1 - EV production and protein quantification A) Microscopic images of Expi293F cells at harvest, three days after transfection. B) Schematic EV processing workflow. C) EVs produced by Expi293F cells were concentrated by TFF and ultrafiltration, followed by size-exclusion chromatography (SEC). Protein concentration on SEC fractions was determined by microBCA. Data from $n=6 \mathrm{EV}$ batches are shown. D) Stain-free imaging of SDS-PAGE on equal volumes of SEC fractions $1-11$, pooled EVs (fr. 1-4) and cell lysate (CL). Image is representative of $n=6 E V$ batches.}

\section{Supplemental Figure 2 - Characterization of palmGRET EVs by imaging flow cytometry Different EV batches were analyzed by imaging flow cytometry. Single-stain controls, free dye control and PBS were included for gating and determination of background signal. A) Gating strategy. Speed-beads were gated out, after which MemGlow and GFP gates were set based on Expi293 EV labelled only with MemGlow (left), and palmGRET-EV not labelled with MemGlow (middle). B) Dilution series of palmGRET-MemGlow EV shows}


the linearity of the measuring range. C) Addition of $1 \%$ Triton results in loss of palmGRETMemGlow double positive objects. D) Percentage of palmGRET+MemGlow positive EVs in $\mathrm{n}=6$ batches, compared with unlabeled EV, PBS, and free dye (all measured twice).

\section{Supplemental Figure 3 - Intrathecal injection of EVs leads to rapid clearance from} CSF A) 3E10 palmGRET EVs were injected intrathecally into a macaque. CSF and plasma were sampled before and $0.5,1,3,6$, and 24 hours afterwards. EVs were detected in CSF and plasma by Nano-Glo assay. Error bars represent the assay variability.

Supplemental Figure 4 - Flow cytometry on PBMCs in whole blood A) Overall gating strategy. First, single cells were gated from the total events. Monocyte and lymphocyte subsets were identified based on FSC / SSC profiles. Monocytes were identified by subsequently gating for CD3-CD159- cells, followed by gating for CD20- cells. Lymphocytes were divided in CD3+ cells, which contained both CD8+ and CD4+ T cells, and CD3- cells which contained both CD3- CD20+ B cells as well as CD3- CD159+ NK cells. B) Analogous to Figure B, quantification of \%GFP and \% MemGlow-positive CD4+ and CD8+ T cells, as percentage of the total CD4+ and CD8+ T cells, respectively. Data from $n=2$ intravenous $E V$ administrations are shown. C) Quantification of \%GFP and \%MemGlow positive CD3CD159+ NK cells as percentage of the total NK cells. Data from $n=2$ intravenous EV administrations are shown. 


\section{References}

1. Lener, T. et al. Applying extracellular vesicles based therapeutics in clinical trials - an ISEV position paper. J Extracell Vesicles 4, 30087 (2015).

2. Allan, D., Tieu, A., Lalu, M. \& Burger, D. Mesenchymal stromal cell-derived extracellular vesicles for regenerative therapy and immune modulation: Progress and challenges toward clinical application. Stem Cells Transl. Med. 9, 39-46 (2020).

3. Escudé Martinez de Castilla, P. et al. Extracellular vesicles as a drug delivery system:A systematic review of preclinical studies. Adv. Drug Deliv. Rev. 175, (2021).

4. Reiner, A. T. et al. Concise review: Developing best-practice models for the therapeutic use of extracellular vesicles. Stem Cells Transl. Med. 6, (2017).

5. Pascucci, L. et al. Paclitaxel is incorporated by mesenchymal stromal cells and released in exosomes that inhibit in vitro tumor growth: A new approach for drug delivery. J. Control. Release 192, 262-270 (2014).

6. De Jong, O. G. et al. Drug Delivery with Extracellular Vesicles: From Imagination to Innovation. Acc. Chem. Res. 52, 1761-1770 (2019).

7. O'Loughlin, A. J. et al. Functional Delivery of Lipid-Conjugated siRNA by Extracellular Vesicles. Mol. Ther. 25, 1580-1587 (2017).

8. Usman, W. M. et al. Efficient RNA drug delivery using red blood cell extracellular vesicles. Nat. Commun. 9, (2018).

9. Kim, S. M. et al. Cancer-derived exosomes as a delivery platform of CRISPR/Cas9 confer cancer cell tropism-dependent targeting. J. Control. Release 266, 8-16 (2017).

10. Yao, X. et al. Engineered extracellular vesicles as versatile ribonucleoprotein delivery vehicles for efficient and safe CRISPR genome editing. J. Extracell. Vesicles 10, (2021).

11. Witwer, K. W. \& Wolfram, J. Extracellular vesicles versus synthetic nanoparticles for drug delivery. Nature Reviews Materials vol. 6 103-106 (2021).

12. Alvarez-Erviti, L. et al. Delivery of siRNA to the mouse brain by systemic injection of targeted exosomes. Nat. Biotechnol. 29, 306-309 (2011).

13. Noren Hooten, N. et al. Hitting the Bullseye: Are extracellular vesicles on target ? J. Extracell. Vesicles (2020) doi:10.1002/jev2.12032.

14. Pham, T. C. et al. Covalent conjugation of extracellular vesicles with peptides and nanobodies for targeted therapeutic delivery. J. Extracell. Vesicles 10, (2021).

15. Zhu, X. et al. Comprehensive toxicity and immunogenicity studies reveal minimal effects in mice following sustained dosing of extracellular vesicles derived from HEK293T cells. J. Extracell. Vesicles 6, (2017).

16. Saleh, A. F. et al. Extracellular vesicles induce minimal hepatotoxicity and immunogenicity. Nanoscale 11, 6990-7001 (2019).

17. Lai, C. P. et al. Dynamic biodistribution of extracellular vesicles in vivo using a multimodal imaging reporter. ACS Nano 8, 483-94 (2014).

18. Wiklander, O. P. B. et al. Extracellular vesicle in vivo biodistribution is determined by cell source, route of administration and targeting. J. Extracell. Vesicles 4, 1-13 (2015).

19. Kooijmans, S. A. A. et al. PEGylated and targeted extracellular vesicles display 
enhanced cell specificity and circulation time. J. Control. Release 224, 77-85 (2016).

20. Betzer, O. et al. In Vivo Neuroimaging of Exosomes Using Gold Nanoparticles. ACS Nano 11, 10883-10893 (2017).

21. Gupta, D. et al. Quantification of extracellular vesicles in vitro and in vivo using sensitive bioluminescence imaging. J. Extracell. Vesicles 9, (2020).

22. Wu, A. Y. T. et al. Multiresolution Imaging Using Bioluminescence Resonance Energy Transfer Identifies Distinct Biodistribution Profiles of Extracellular Vesicles and Exomeres with Redirected Tropism. Adv. Sci. 2001467, 1-17 (2020).

23. Lázaro-lbañez, E. et al. Selection of Fluorescent, Bioluminescent, and Radioactive Tracers to Accurately Reflect Extracellular Vesicle Biodistribution in Vivo. ACS Nano (2021) doi:10.1021/acsnano.0c09873.

24. Leenaars, C. H. C. et al. Animal to human translation: A systematic scoping review of reported concordance rates. J. Transl. Med. 17, 1-22 (2019).

25. Ophelders, D. et al. Mesenchymal Stromal Cell-Derived Extracellular Vesicles Protect the Fetal Brain After Hypoxia-Ischemia. Stem Cells Transl. Med. 754-763 (2016).

26. Potz, B. A. et al. Extracellular vesicle injection improves myocardial function and increases angiogenesis in a swine model of chronic ischemia. J. Am. Heart Assoc. 7, (2018).

27. Kang, M., Jordan, V., Blenkiron, C. \& Chamley, L. W. Biodistribution of extracellular vesicles following administration into animals: A systematic review. J. Extracell. Vesicles 10, (2021).

28. Collot, M. et al. MemBright: A Family of Fluorescent Membrane Probes for Advanced Cellular Imaging and Neuroscience. Cell Chem. Biol. 26, 600-614.e7 (2019).

29. Hyenne, V. et al. Studying the Fate of Tumor Extracellular Vesicles at High Spatiotemporal Resolution Using the Zebrafish Embryo. Dev. Cell 48, 554-572.e7 (2019).

30. Welsh, J. A. et al. MIFlowCyt-EV: a framework for standardized reporting of extracellular vesicle flow cytometry experiments. J. Extracell. Vesicles 9, (2019).

31. Van Deun, J. et al. EV-TRACK: transparent reporting and centralizing knowledge in extracellular vesicle research. Nat. Methods 14, 228-232 (2017).

32. Théry, C. et al. Minimal information for studies of extracellular vesicles 2018 (MISEV2018): a position statement of the International Society for Extracellular Vesicles and update of the MISEV2014 guidelines. J. Extracell. Vesicles 7, 1535750 (2018).

33. Erdbrügger, U. et al. Imaging flow cytometry elucidates limitations of microparticle analysis by conventional flow cytometry. Cytometry. A 85, 756-70 (2014).

34. Görgens, A. et al. Optimisation of imaging flow cytometry for the analysis of single extracellular vesicles by using fluorescence-tagged vesicles as biological reference material. J. Extracell. Vesicles 8, (2019).

35. Morishita, M. et al. Quantitative analysis of tissue distribution of the B16BL6-derived exosomes using a streptavidin-lactadherin fusion protein and lodine-125-Labeled biotin derivative after intravenous injection in mice. J. Pharm. Sci. 104, 705-713 (2015).

36. Verweij, F. J. et al. Live Tracking of Inter-organ Communication by Endogenous 
Exosomes In Vivo. Dev. Cell 48, 573-589.e4 (2019).

37. Su, Y. et al. Novel NanoLuc substrates enable bright two-population bioluminescence imaging in animals. Nat. Methods 17, 852-860 (2020).

38. Takeuchi, $\mathrm{Y}$. et al. Type $\mathrm{C}$ retrovirus inactivation by human complement is determined by both the viral genome and the producer cell. J. Virol. 68, 8001-8007 (1994).

39. DePolo, N. J. et al. The Resistance of Retroviral Vectors Produced from Human Cells to Serum Inactivation In Vivo and In Vitro Is Primate Species Dependent. J. Virol. 73, 6708-6714 (1999).

40. Takeuchi, Y. et al. Sensitization of cells and retroviruses to human serum by (a1-3) galactosyltransferase. Nature 379, 85-88 (1996).

41. Chiarelli, P. et al. Nanoparticle biokinetics in mice and nonhuman primates. ACS Nano 11, 9514-9524 (2017).

42. Igarashi, T. et al. Human immunodeficiency virus type 1 neutralizing antibodies accelerate clearance of cell-free virions from blood plasma. Nat. Med. 5, 211-216 (1999).

43. Somiya, M., Yoshioka, Y. \& Ochiya, T. Biocompatibility of highly purified bovine milkderived extracellular vesicles. J. Extracell. Vesicles 7, 1440132 (2018).

44. Urzì, O., Raimondo, S. \& Alessandro, R. Extracellular vesicles from plants: Current knowledge and open questions. Int. J. Mol. Sci. 22, (2021).

45. Dams, E. T. M. et al. Accelerated blood clearance and altered biodistribution of repeated injections of sterically stabilized liposomes. J. Pharmacol. Exp. Ther. 292, 1071-1079 (2000).

46. Ishida, T. et al. Injection of PEGylated liposomes in rats elicits PEG-specific IgM, which is responsible for rapid elimination of a second dose of PEGylated liposomes. J. Control. Release 112, 15-25 (2006).

47. Wedege, E. \& Frøholm, L. O. Human antibody response to a group B serotype 2a meningococcal vaccine studied by an immunoblotting technique. Antonie Van Leeuwenhoek 52, 265-266 (1986).

48. Zitvogel, L. et al. Eradication of established murine tumors using a novel cell-free vaccine: Dendritic cell-derived exosomes. Nat. Med. 4, 594-600 (1998).

49. Kim, O. Y. et al. Immunization with Escherichia coli Outer Membrane Vesicles Protects Bacteria - Induced Lethality via Th1 and Th17 Cell Responses . J. Immunol. 190, 4092-4102 (2013).

50. Zeelenberg, I. S. et al. Targeting tumor antigens to secreted membrane vesicles in vivo induces efficient antitumor immune responses. Cancer Res. 68, 1228-1235 (2008).

51. Liu, Q. et al. Outer membrane vesicles derived from Salmonella Typhimurium mutants with truncated LPS induce cross-protective immune responses against infection of Salmonella enterica serovars in the mouse model. Int. J. Med. Microbiol. 306, 697706 (2016).

52. Bliss, C. M. et al. Targeting Antigen to the Surface of EVs Improves the In Vivo Immunogenicity of Human and Non-human Adenoviral Vaccines in Mice. Mol. Ther. Methods Clin. Dev. 16, 108-125 (2020).

53. Jiang, L. et al. A bacterial extracellular vesicle-based intranasal vaccine against 
SARS-CoV-2. bioRxiv (2021).

54. Martínez-Riaño, A. et al. Antigen phagocytosis by $B$ cells is required for a potent humoral response. EMBO Rep. 19, 1-15 (2018).

55. Eitan, E. et al. Age-Related Changes in Plasma Extracellular Vesicle Characteristics and Internalization by Leukocytes. Sci. Rep. 7, (2017).

56. Imai, T. et al. Macrophage-dependent clearance of systemically administered B16BL6-derived exosomes from the blood circulation in mice. J. Extracell. vesicles 4, 1-8 (2015).

57. Watson, D. C. et al. Efficient production and enhanced tumor delivery of engineered extracellular vesicles. Biomaterials 105, 195-205 (2016).

58. Praetorius, J. \& Damkier, H. H. Transport across the choroid plexus epithelium. Am. J. Physiol. - Cell Physiol. 312, C673-C686 (2017).

59. Schwartz, M. W. et al. Evidence for entry of plasma insulin into cerebrospinal fluid through an intermediate compartment in dogs. Quantitative aspects and implications for transport. J. Clin. Invest. 88, 1272-1281 (1991).

60. Zhuang, X. et al. Treatment of brain inflammatory diseases by delivering exosome encapsulated anti-inflammatory drugs from the nasal region to the brain. Mol. Ther. 19, 1769-1779 (2011).

61. Hu, G. et al. Astrocyte EV-Induced lincRNA-Cox2 Regulates Microglial Phagocytosis: Implications for Morphine-Mediated Neurodegeneration. Mol. Ther. - Nucleic Acids 13, 450-463 (2018).

62. Thomi, G. et al. Intranasally Administered Exosomes from Umbilical Cord Stem Cells Have Preventive Neuroprotective Effects and Contribute to Functional Recovery after Perinatal Brain Injury. Cells 8, (2019).

63. Upadhya, R. et al. Extracellular vesicles from human iPSC-derived neural stem cells: miRNA and protein signatures, and anti-inflammatory and neurogenic properties. $J$. Extracell. Vesicles 9, (2020).

64. Hayes, S. H. et al. Brain Targeting and Toxicological Assessment of the Extracellular Vesicle - Packaged Antioxidant Catalase - SKL Following Intranasal Administration in Mice. (2021) doi:10.1007/s12640-021-00390-6.

65. Pathipati, P. et al. Mesenchymal Stem Cell ( MSC )- Derived Extracellular Vesicles Protect from Neonatal Stroke by Interacting with Microglial Cells. Neurotherapeutics (2021) doi:10.1007/s13311-021-01076-9.

66. Johnson, J. E. et al. Neurovirulence properties of recombinant vesicular stomatitis virus vectors in non-human primates. Virology 360, 36-49 (2007).

67. Fukuyama, Y. et al. Nanogel-based pneumococcal surface protein A nasal vaccine induces microRNA-associated Th17 cell responses with neutralizing antibodies against Streptococcus pneumoniae in macaques. Mucosal Immunol. 8, 1144-1153 (2015).

68. Southam, D. S., Dolovich, M., O’Byrne, P. M. \& Inman, M. D. Distribution of intranasal instillations in mice: Effects of volume, time, body position, and anesthesia. Am. J. Physiol. - Lung Cell. Mol. Physiol. 282, 833-839 (2002).

69. Luo, W. et al. Spatial and temporal tracking of cardiac exosomes in mouse using a nano-luciferase-CD63 fusion protein. Commun. Biol. 1-9 doi:10.1038/s42003-0200830-7. 
70. Grange, C. et al. Biodistribution of mesenchymal stem cell-derived extracellular vesicles in a model of acute kidney injury monitored by optical imaging. Int. J. Mol. Med. 33, 1055-1063 (2014).

71. Han, Z. et al. Highly efficient magnetic labelling allows MRI tracking of the homing of stem cell-derived extracellular vesicles following systemic delivery. J. Extracell. Vesicles 10, (2021). 
Figure 1 - Characterization of palmBRET EVs

A
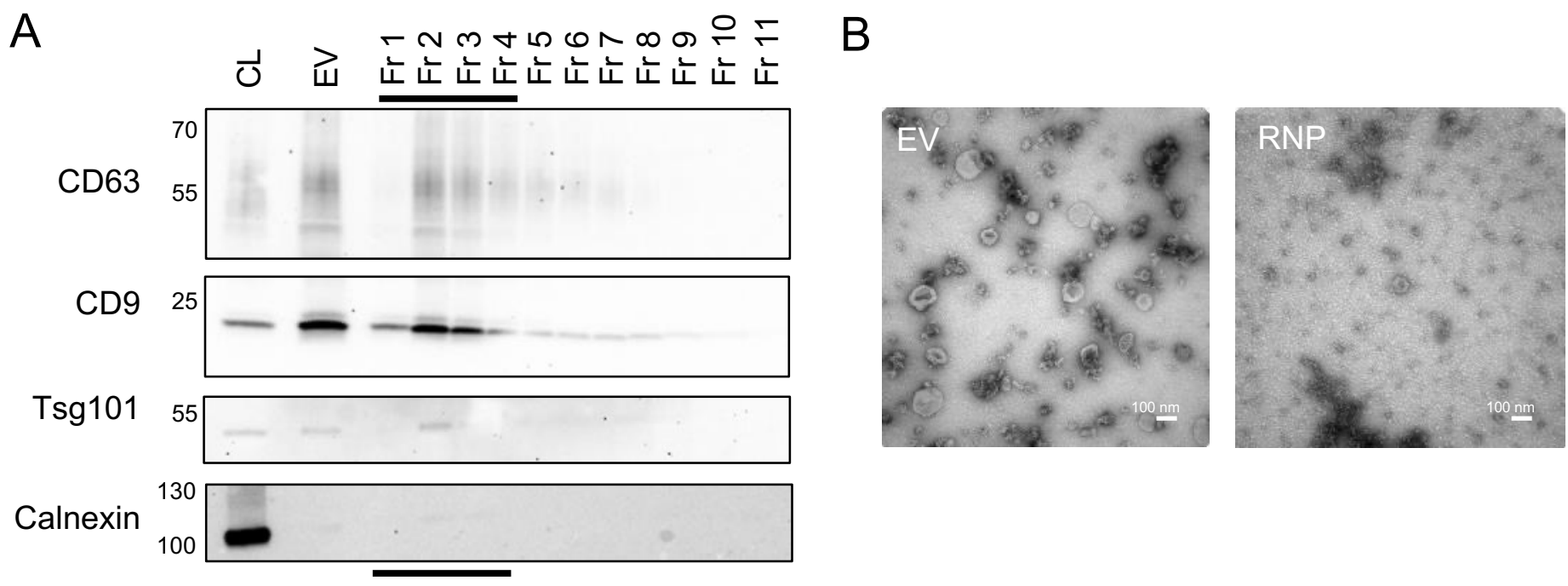

EV

C
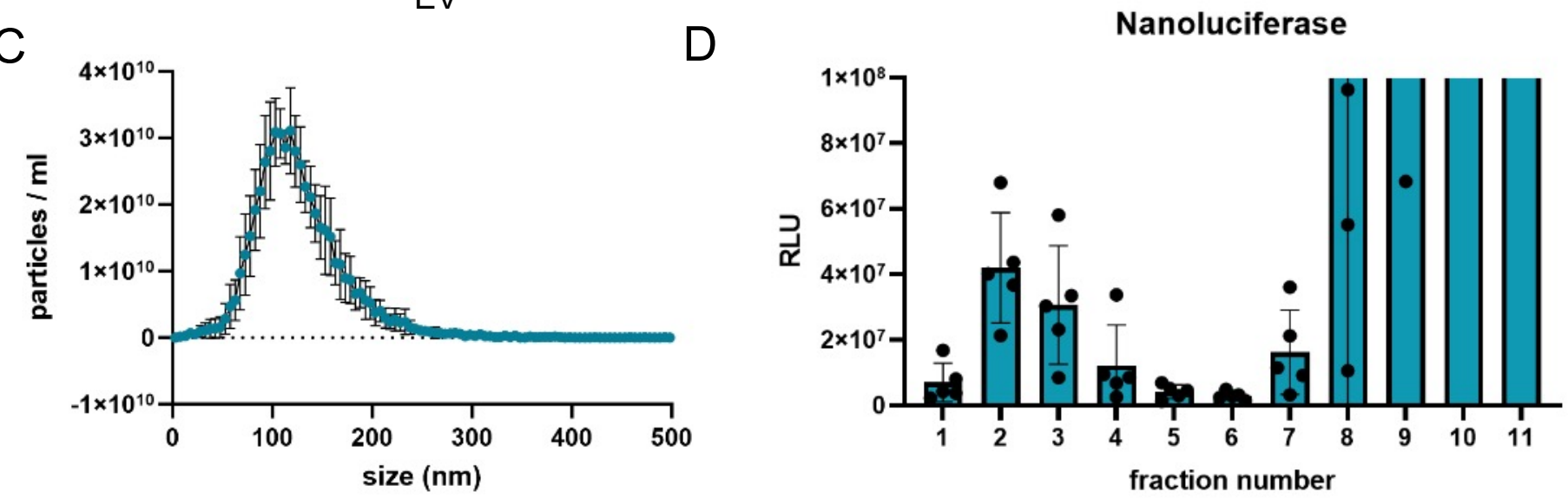

E
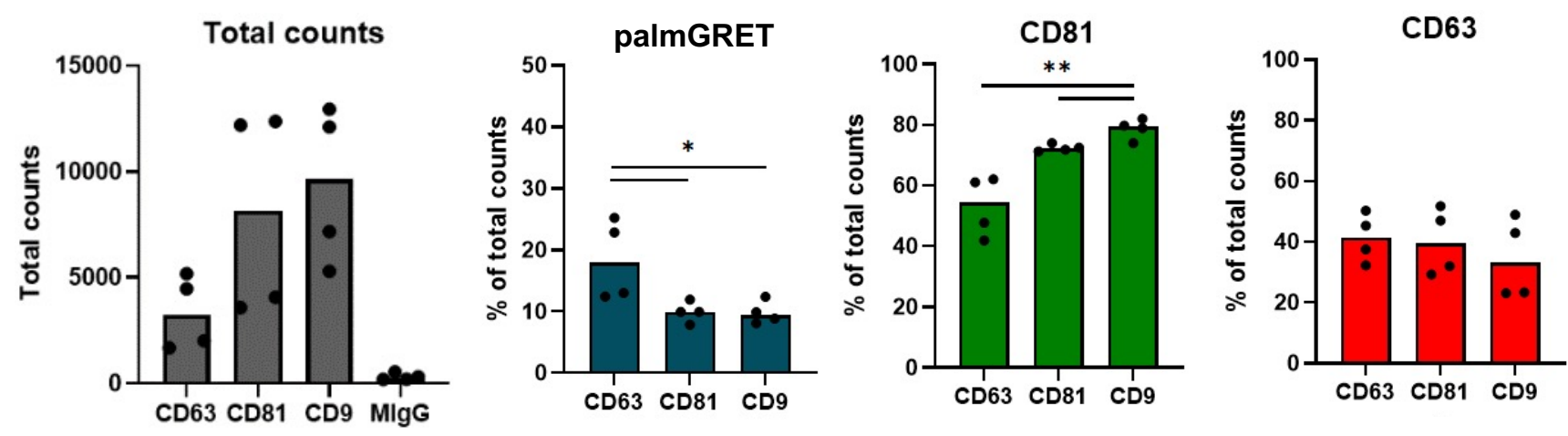

F

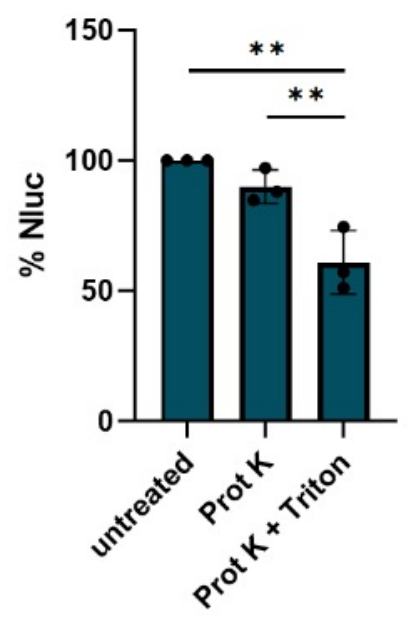

G

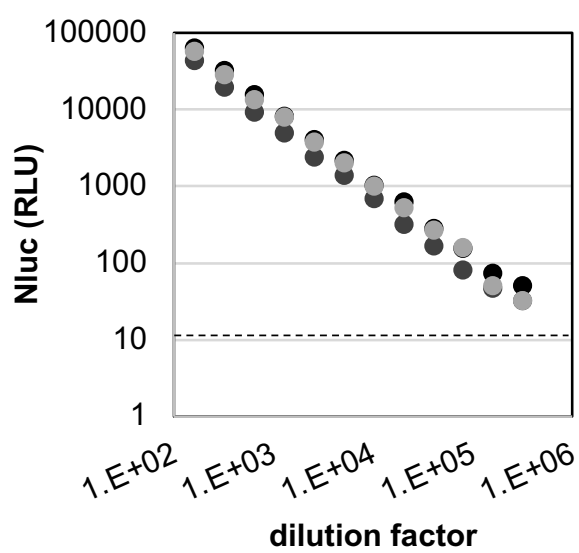

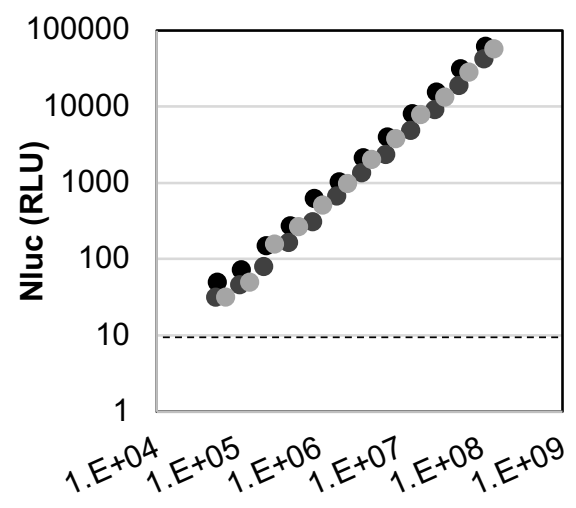

EV number 
Figure 3 - EV uptake by PBMC subsets quantified by flow cytometry

A
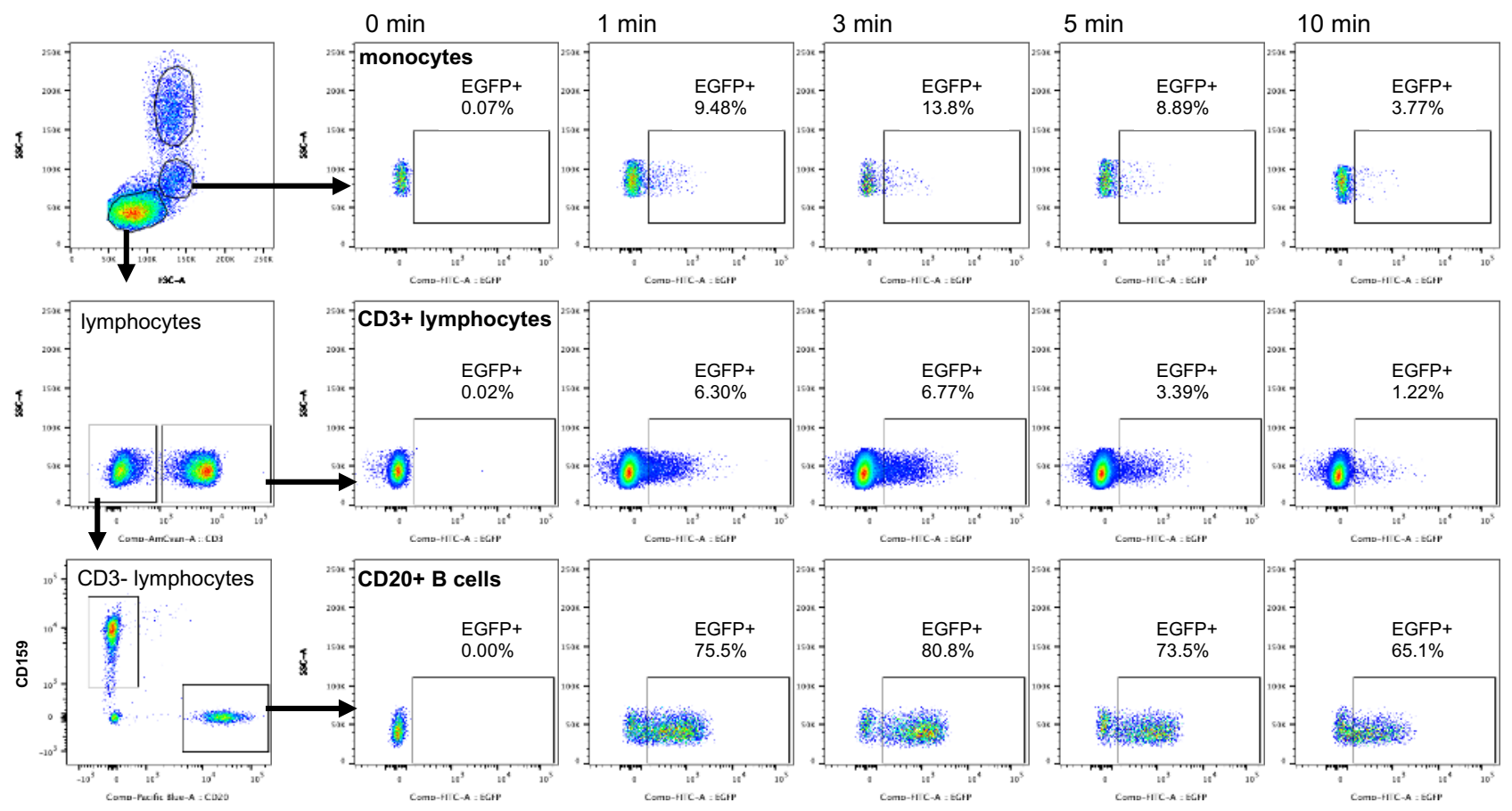

B
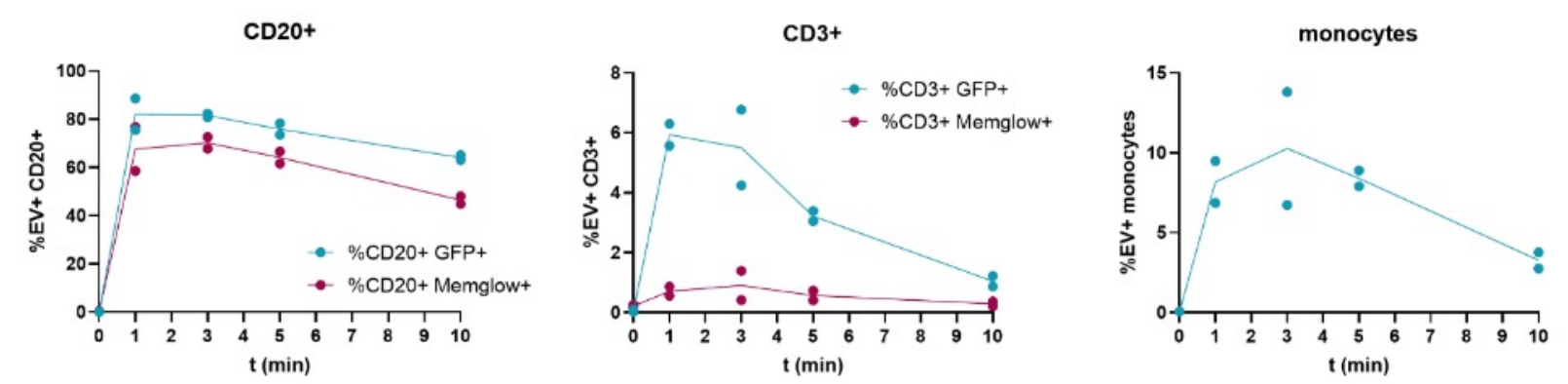

C

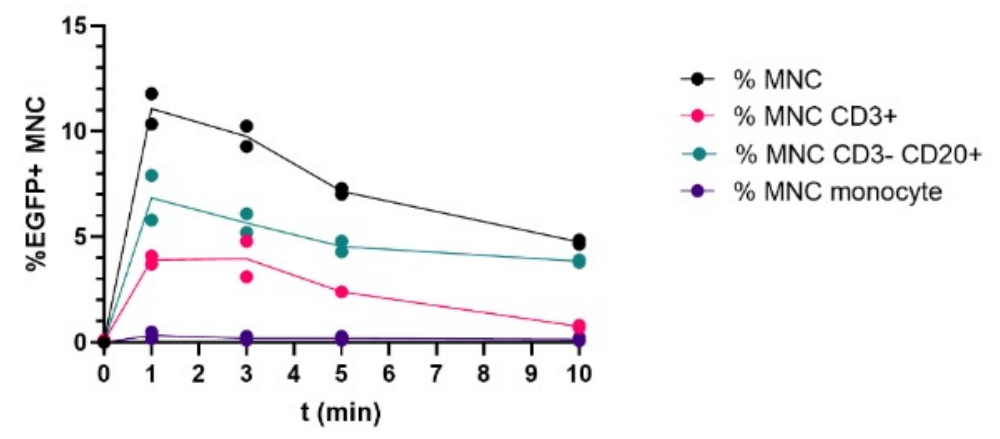


Figure 4 - Biodistribution of palmGRET EVs administered IV or IN into mice

A

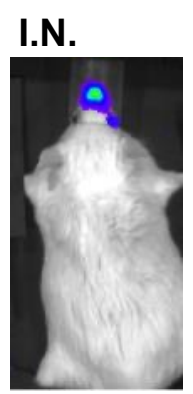

$10 \min 10 \mathrm{~min}$
I.N.

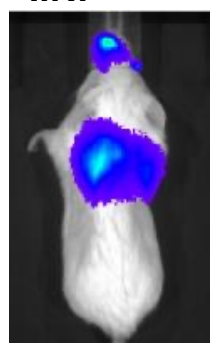

I.V.

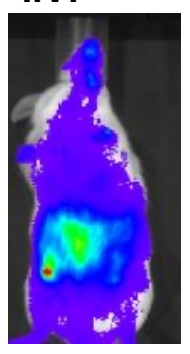

$16 \min$
Ctrl

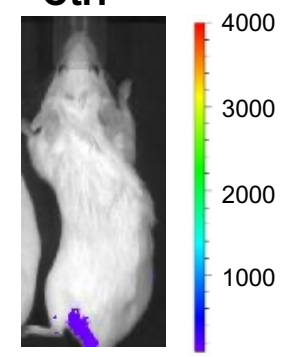

Min $=90$

Max $=4000$

B

I.N.

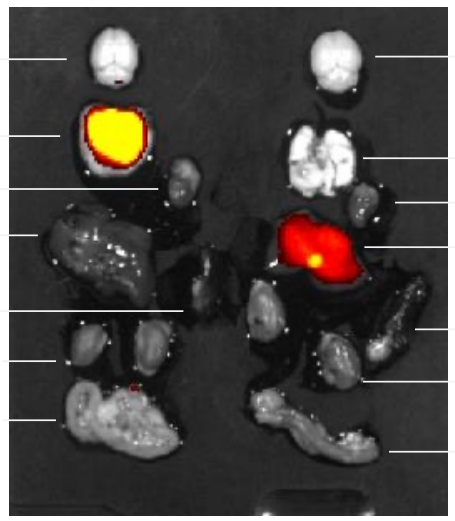

I.V.

Brain
Lung
Heart
Liver
Spleen
Kidneys
Colon

Ctrl.

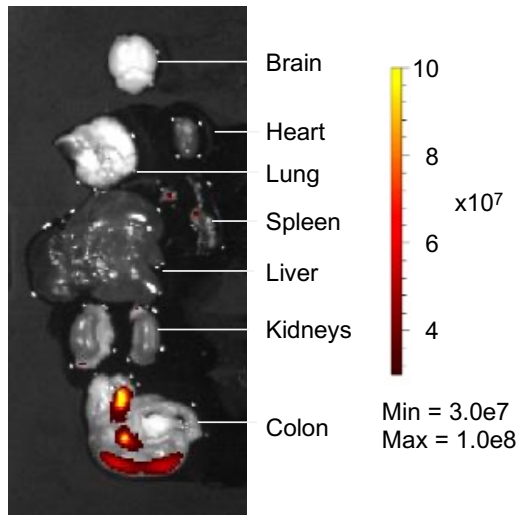

C
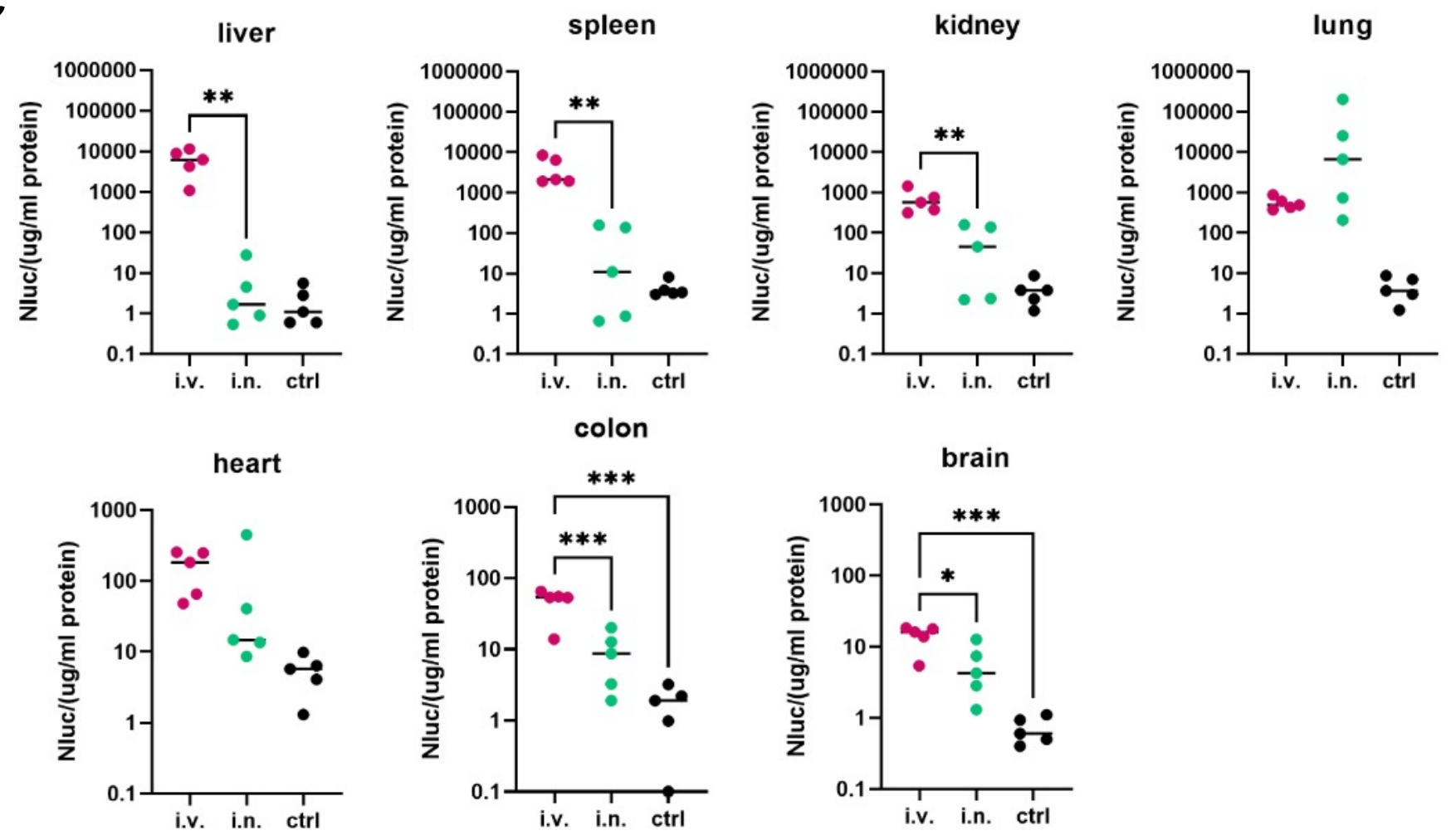
Figure 5 - Biodistribution of palmGRET EVs administered IV or IN into macaques

A

liver

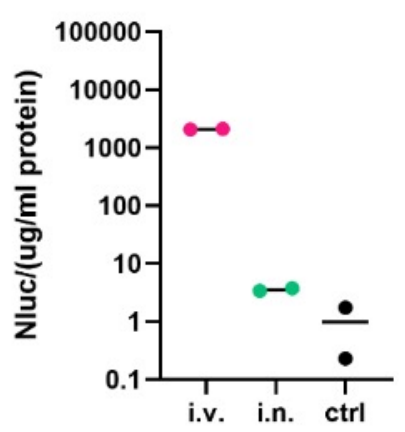

heart

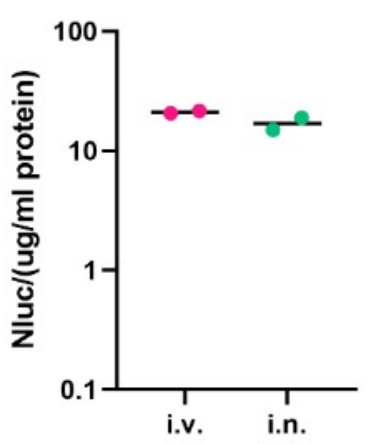

spleen

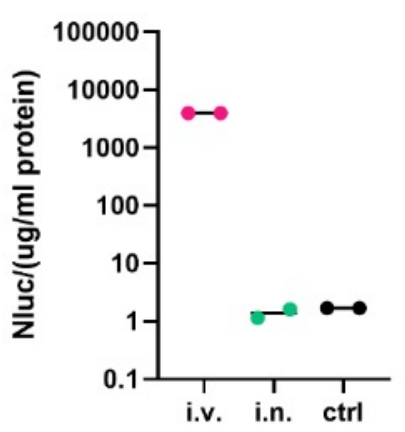

colon

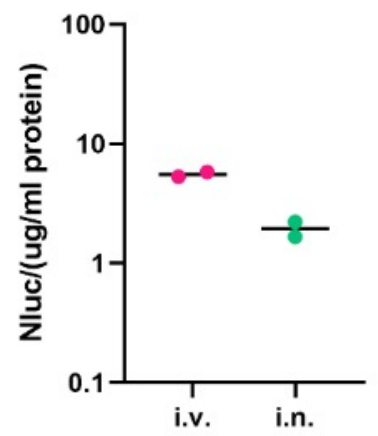

B

intravenous
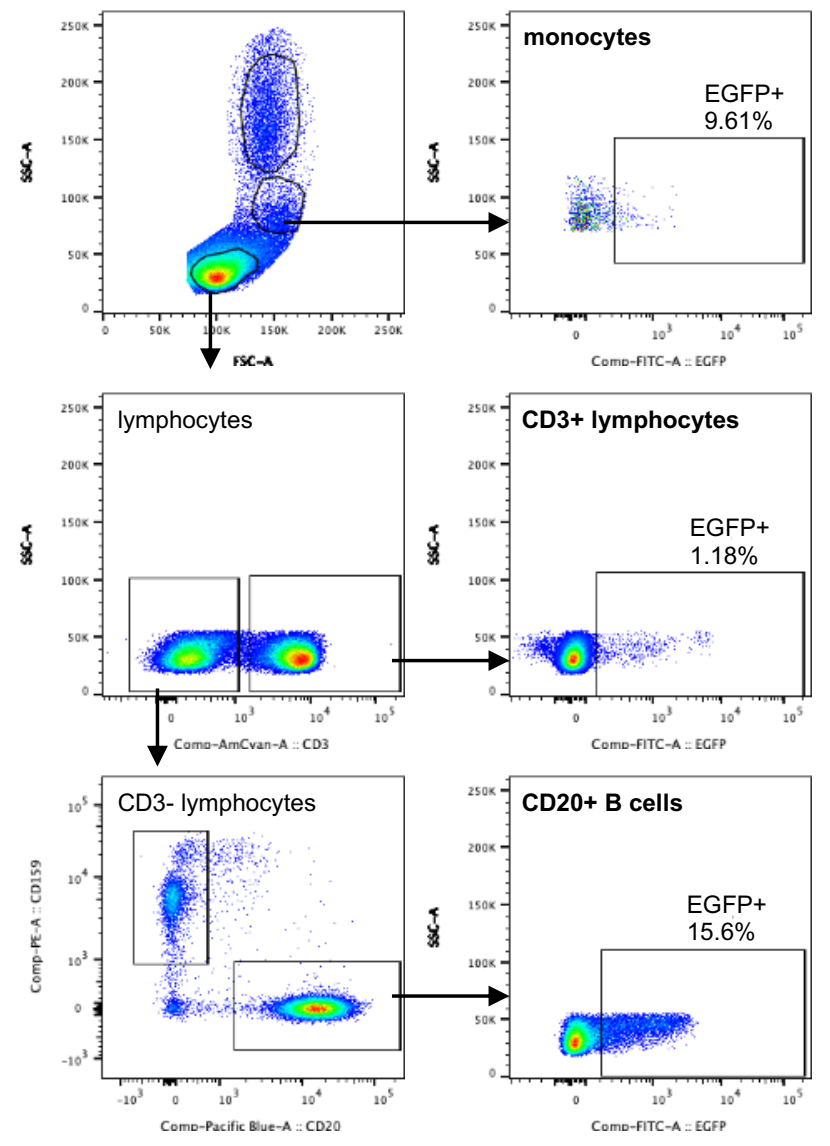

kidney

lung
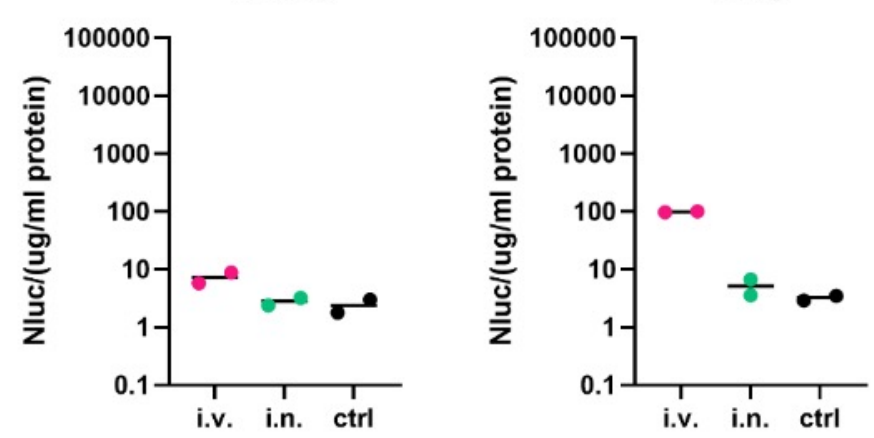

brain

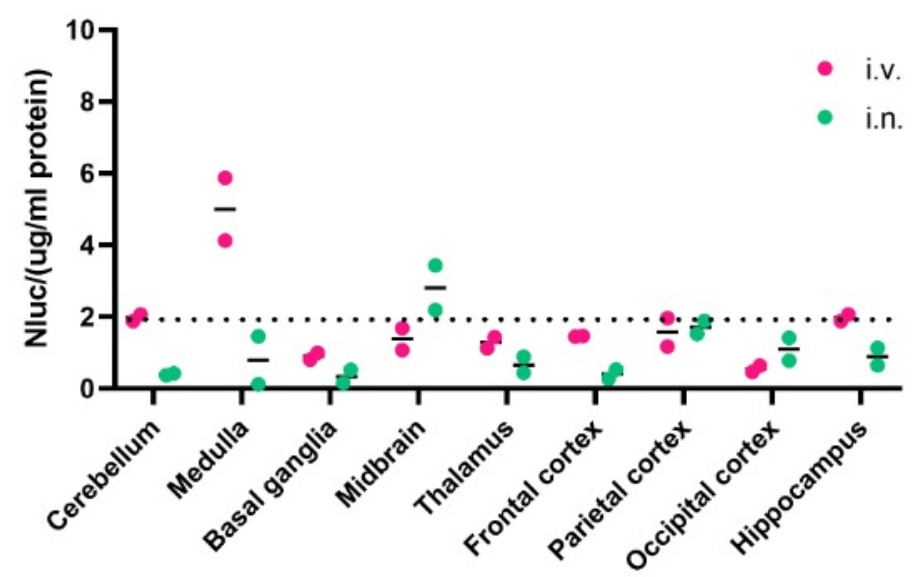

C

intranasal
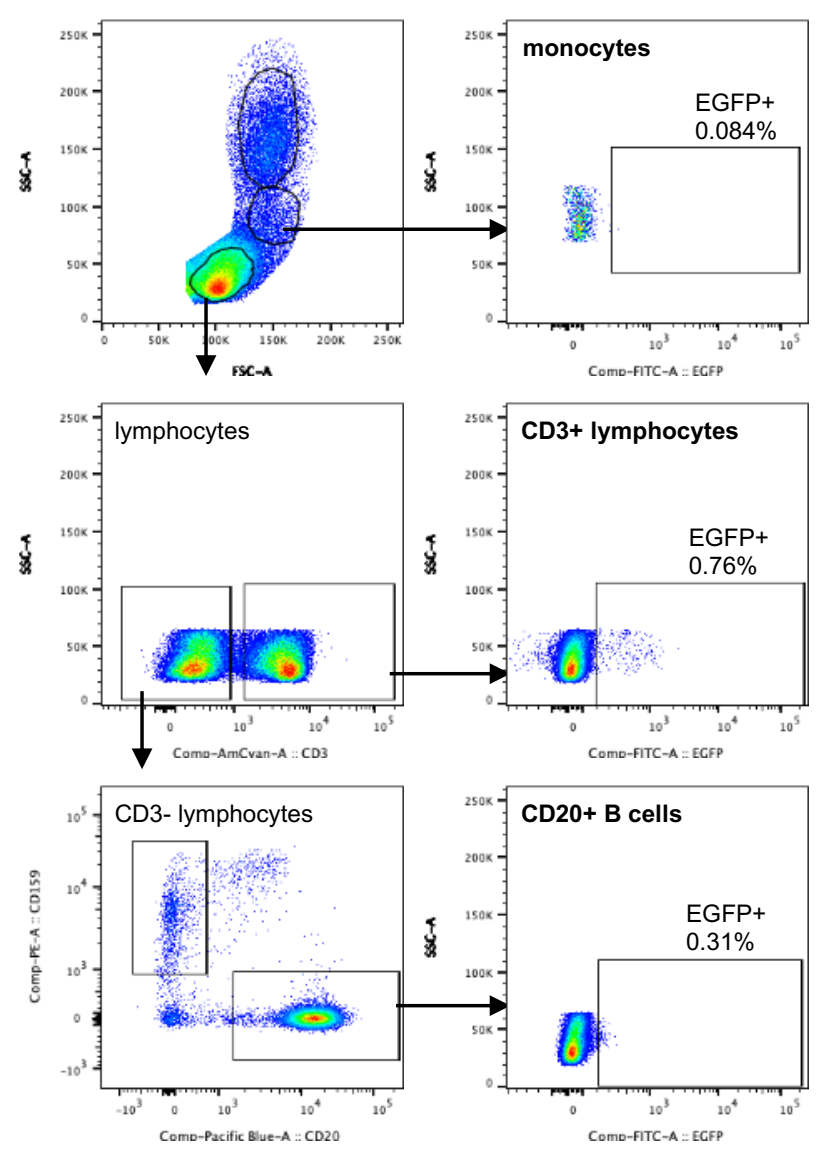


\section{Supplemental Figure 1 - EV production and protein quantification}

A

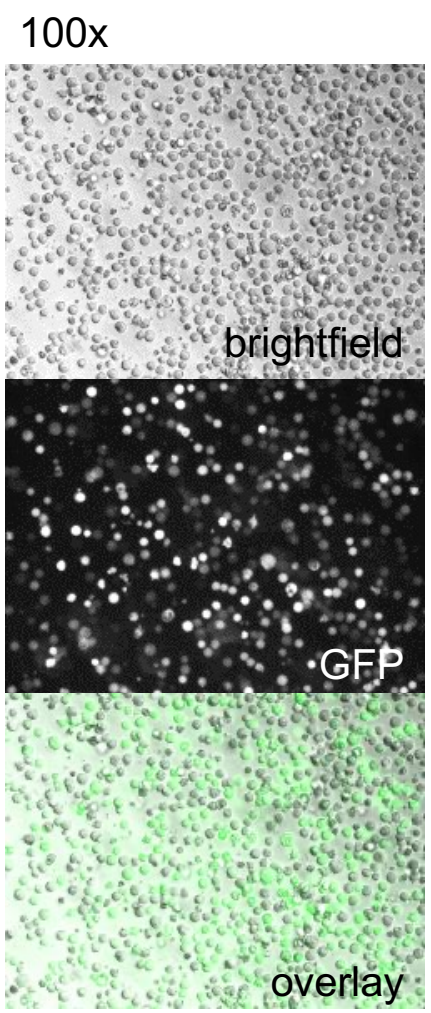

$400 x$

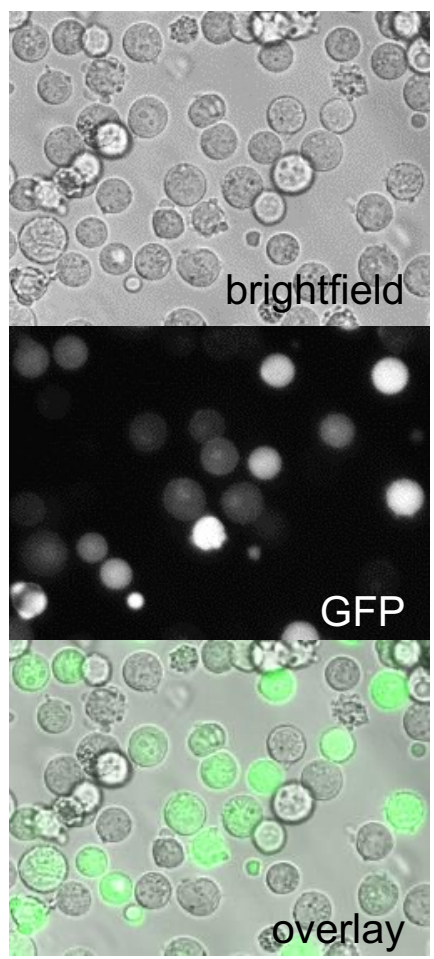

C

microBCA

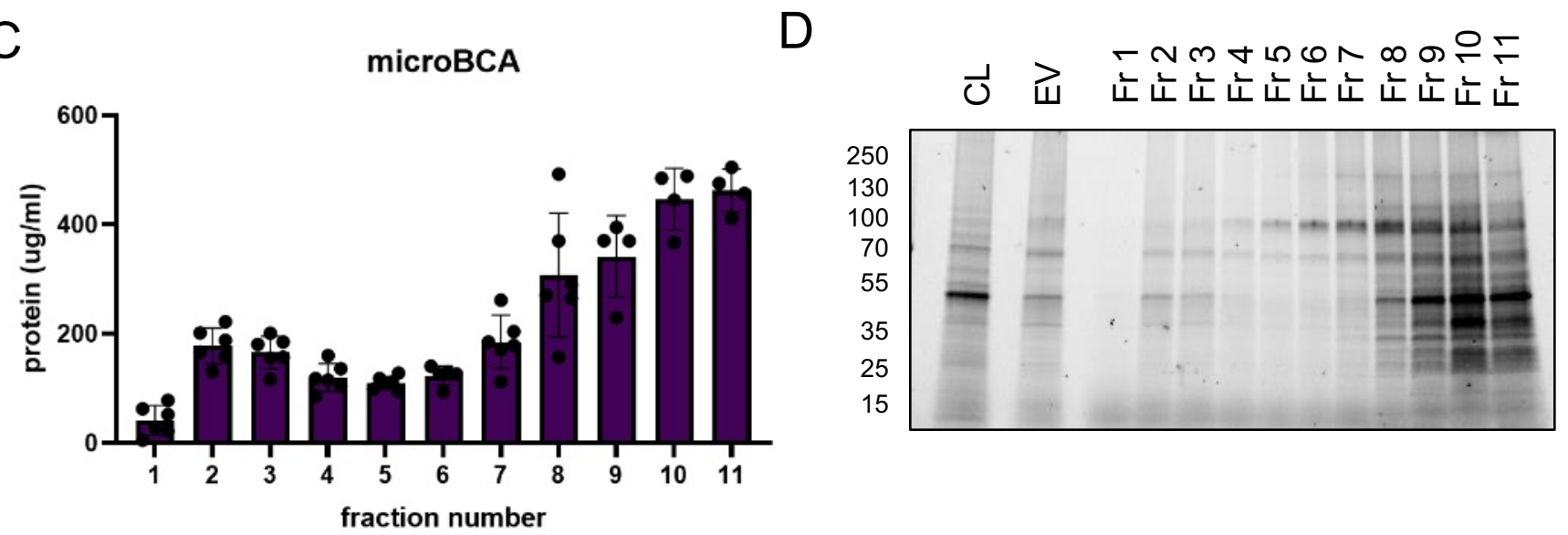

B

$$
\begin{gathered}
\text { ऽ } 20 \mathrm{~min} 1000 \mathrm{~g} \\
20 \mathrm{~min} 2000 \mathrm{~g} \\
0.22 \mu \mathrm{m} \text { filter } \\
\downarrow \\
\text { Concentrate 10x } \\
\text { Vivaflow 50R TFF }
\end{gathered}
$$

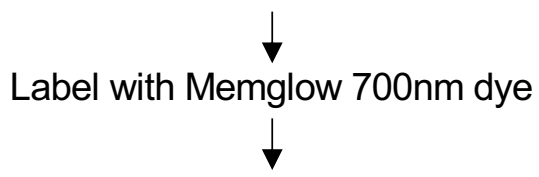

Concentrate on 100KD filter

\begin{tabular}{|c|c|c|c|c|c|c|c|c|}
\hline EV batch nr & 1 & $\underline{2}$ & 3 & 4 & 5 & 6 & average & SD \\
\hline cell dens harvest $(\mathrm{c} / \mathrm{ml})$ & $3.42 \mathrm{E}+06$ & $4.50 \mathrm{E}+06$ & $4.80 \mathrm{E}+06$ & $3.00 \mathrm{E}+06$ & $5.10 \mathrm{E}+06$ & $5.70 \mathrm{E}+06$ & $4.42 \mathrm{E}+06$ & $1.03 E+06$ \\
\hline$\%$ viability & $93.00 \%$ & $90 \%$ & $82 \%$ & $87 \%$ & $89 \%$ & $86 \%$ & $87.83 \%$ & $3.76 \%$ \\
\hline EV protein conc $(\mathrm{ug} / \mathrm{ml})$ & 99 & 140.7 & 305.3 & 157.8 & 234 & 388.9 & 220.95 & 110.27 \\
\hline EV Nluc in (RLU / $50 \mu \mathrm{l})$ & $1.44 \mathrm{E}+06$ & $2.20 \mathrm{E}+07$ & $6.27 \mathrm{E}+07$ & $6.58 \mathrm{E}+07$ & $5.68 \mathrm{E}+07$ & $5.28 \mathrm{E}+07$ & $4.4 \mathrm{E}+07$ & $2.59 \mathrm{E}+07$ \\
\hline EV conc $(\mathrm{p} / \mathrm{ml})$ & $1.60 \mathrm{E}+11$ & $4.40 \mathrm{E}+11$ & $6.00 \mathrm{E}+11$ & $4.50 \mathrm{E}+11$ & $5.70 \mathrm{E}+11$ & $7.60 \mathrm{E}+11$ & $5.0 \mathrm{E}+11$ & $2.02 \mathrm{E}+11$ \\
\hline particle/protein ratio & $1.62 \mathrm{E}+09$ & $3.13 \mathrm{E}+09$ & $1.97 \mathrm{E}+09$ & $2.85 \mathrm{E}+09$ & $2.44 \mathrm{E}+09$ & $1.95 \mathrm{E}+09$ & $2.3 \mathrm{E}+09$ & $5.84 \mathrm{E}+08$ \\
\hline
\end{tabular}

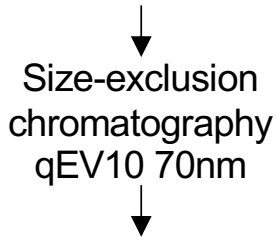

Concentrate on 100KD filter

Aliquot, store at $-80^{\circ} \mathrm{C}$

Supplementary Table 1 - EV production and characterization parameters 
Supplemental Figure 2 - Imagestream of palmGRET EVs by imaging flow cytometry
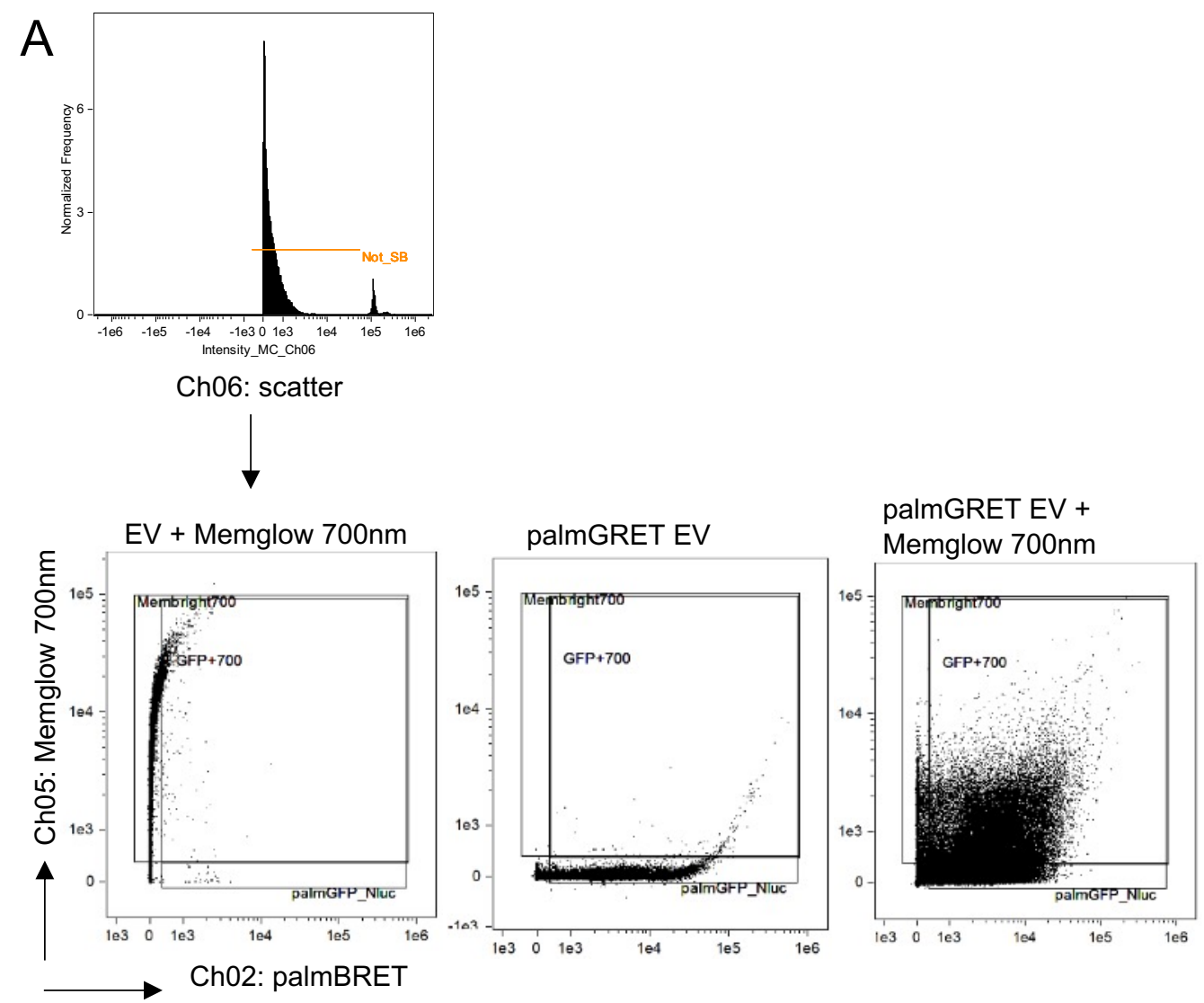

B palmGRET + Memglow 700nm

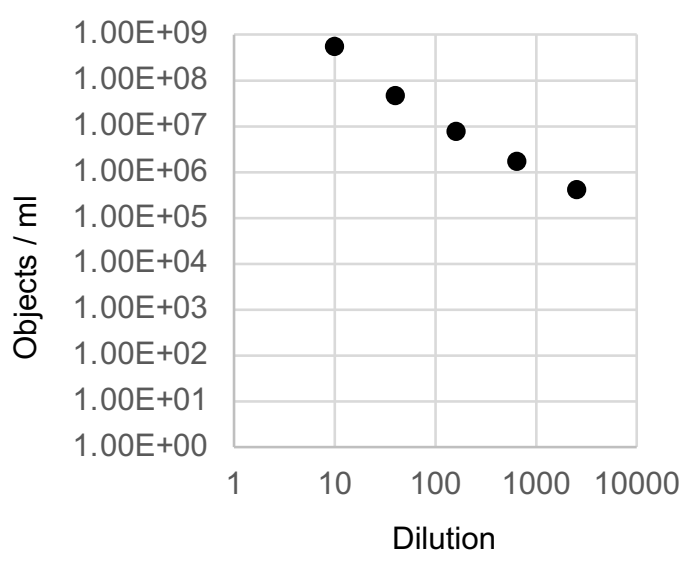

C

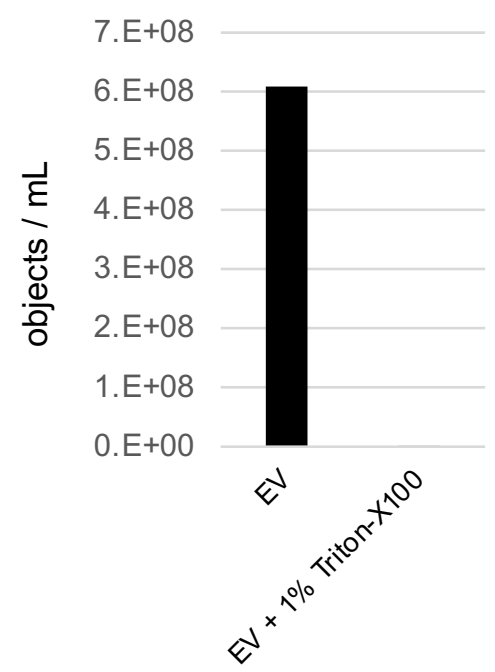

D

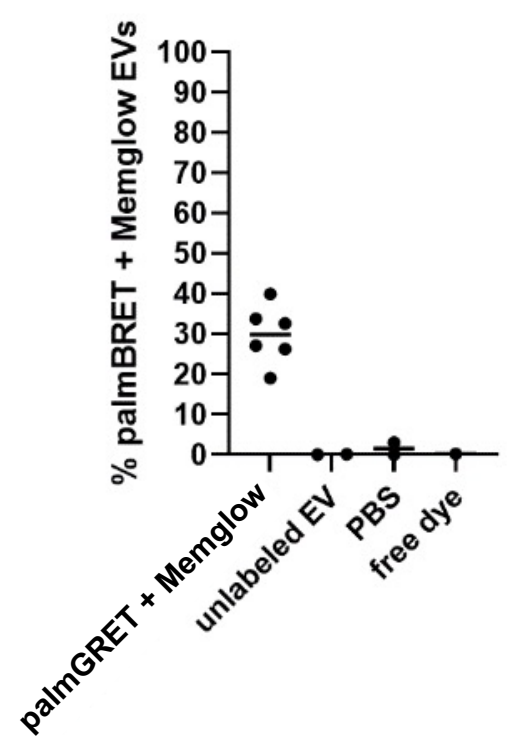


Supplemental Figure 3 - Intrathecal injection leads to rapid clearance of EVs from CSF

Nluc CSF

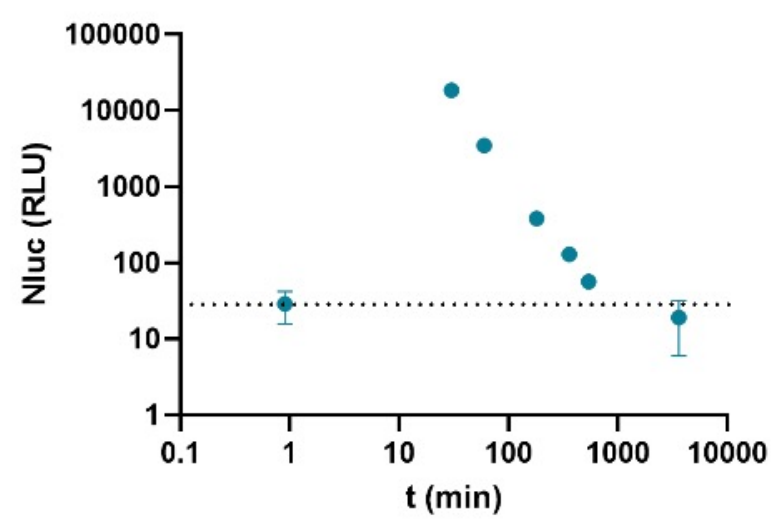

Nluc plasma

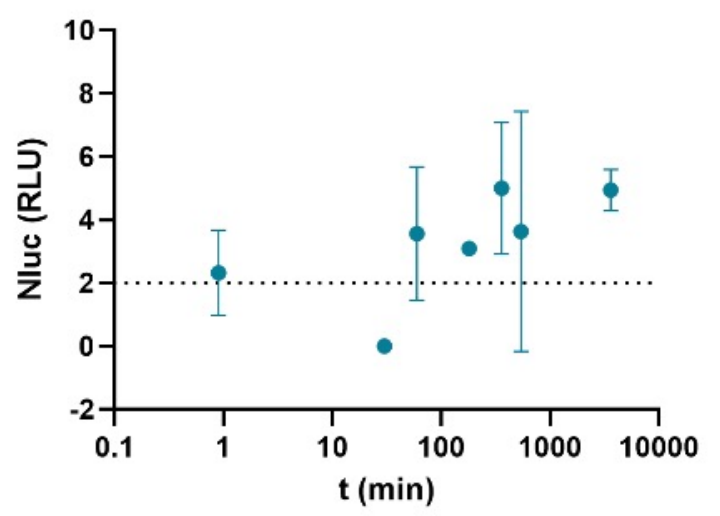

- dose: $3.2 \mathrm{E} 10$ 
Supplementary Figure 4 - Gating strategy of PBMCs quantified by flow cytometry in whole blood

A
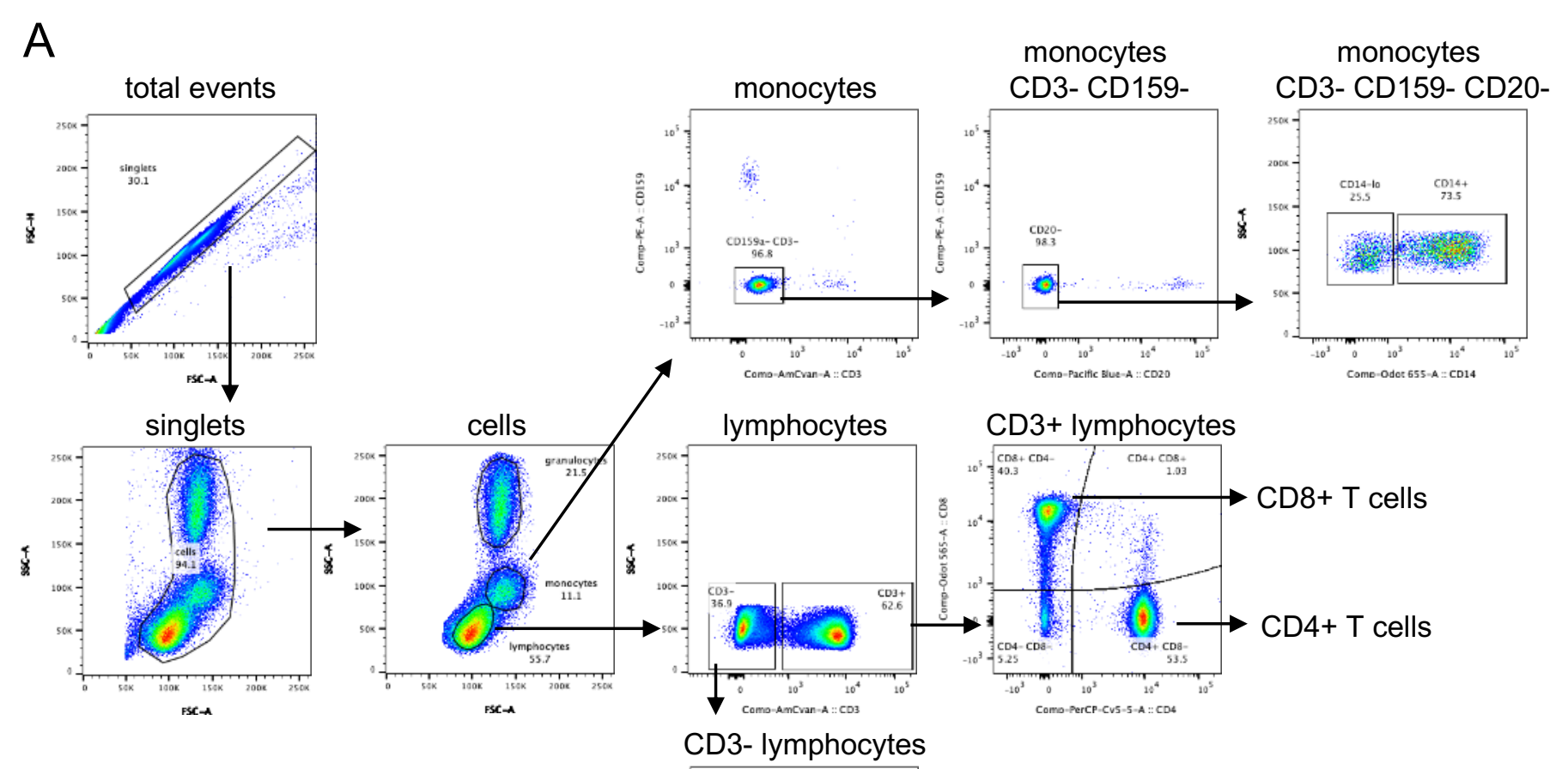

B
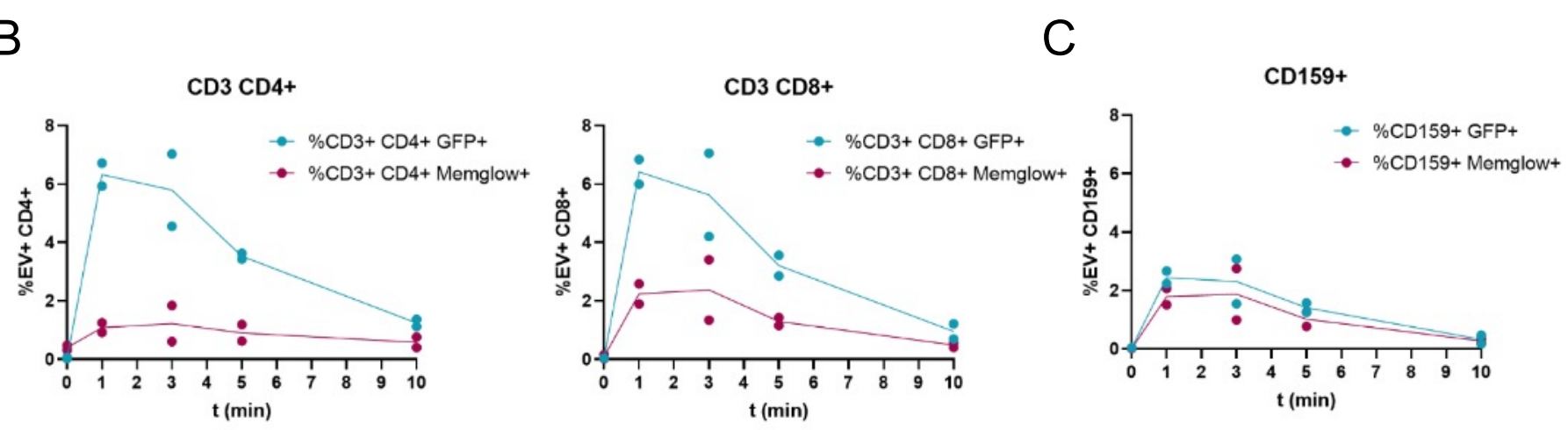

C

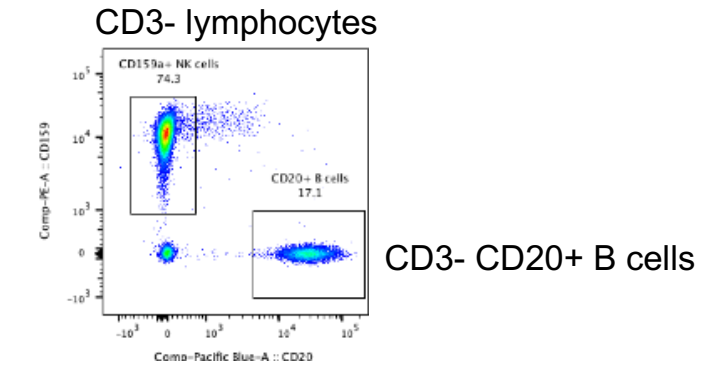

lymphocytes CD3+ lymphocytes

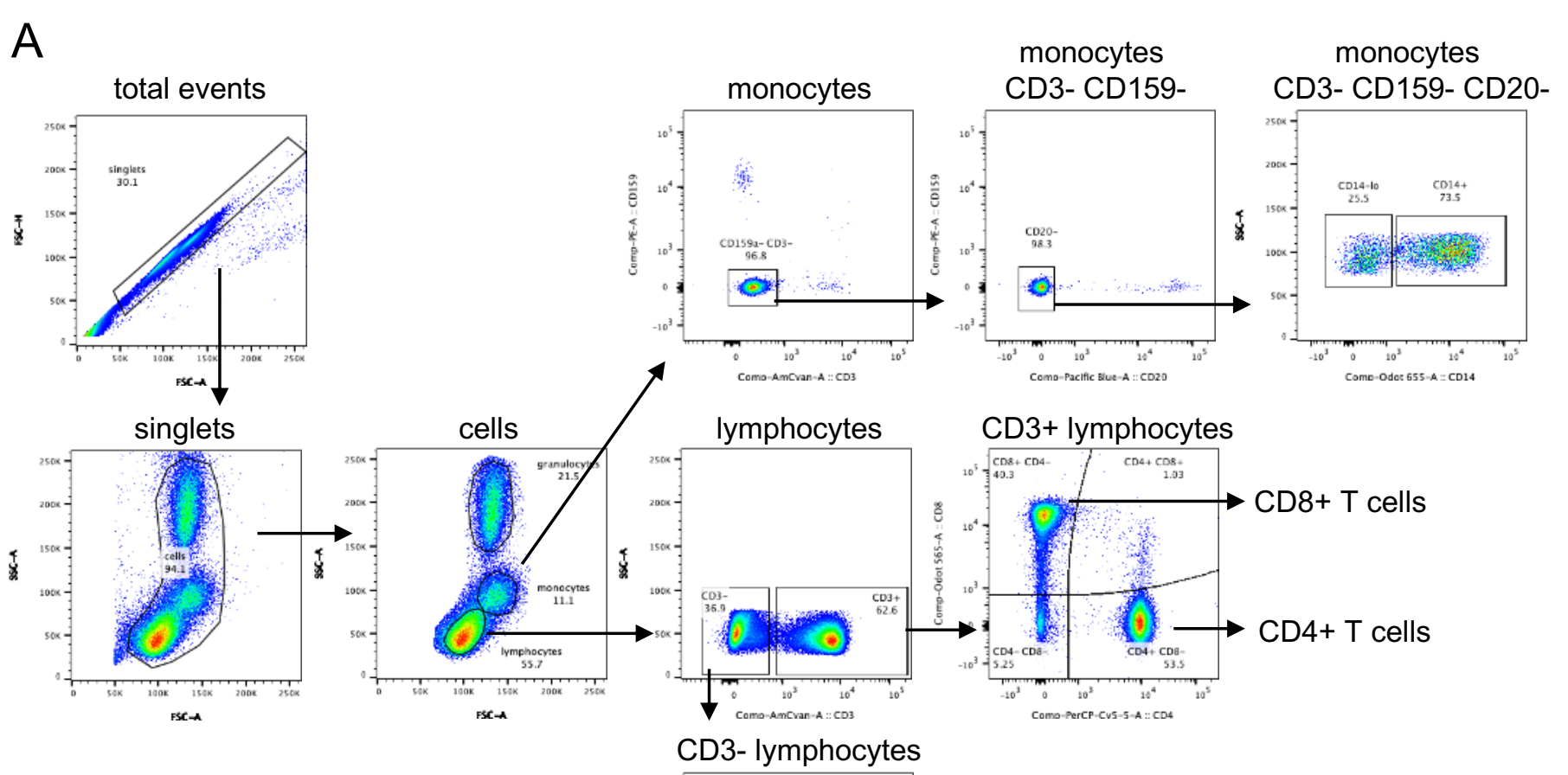

CD3- lymphocytes CD3- CD159-

monocytes CD3- CD159- CD20-

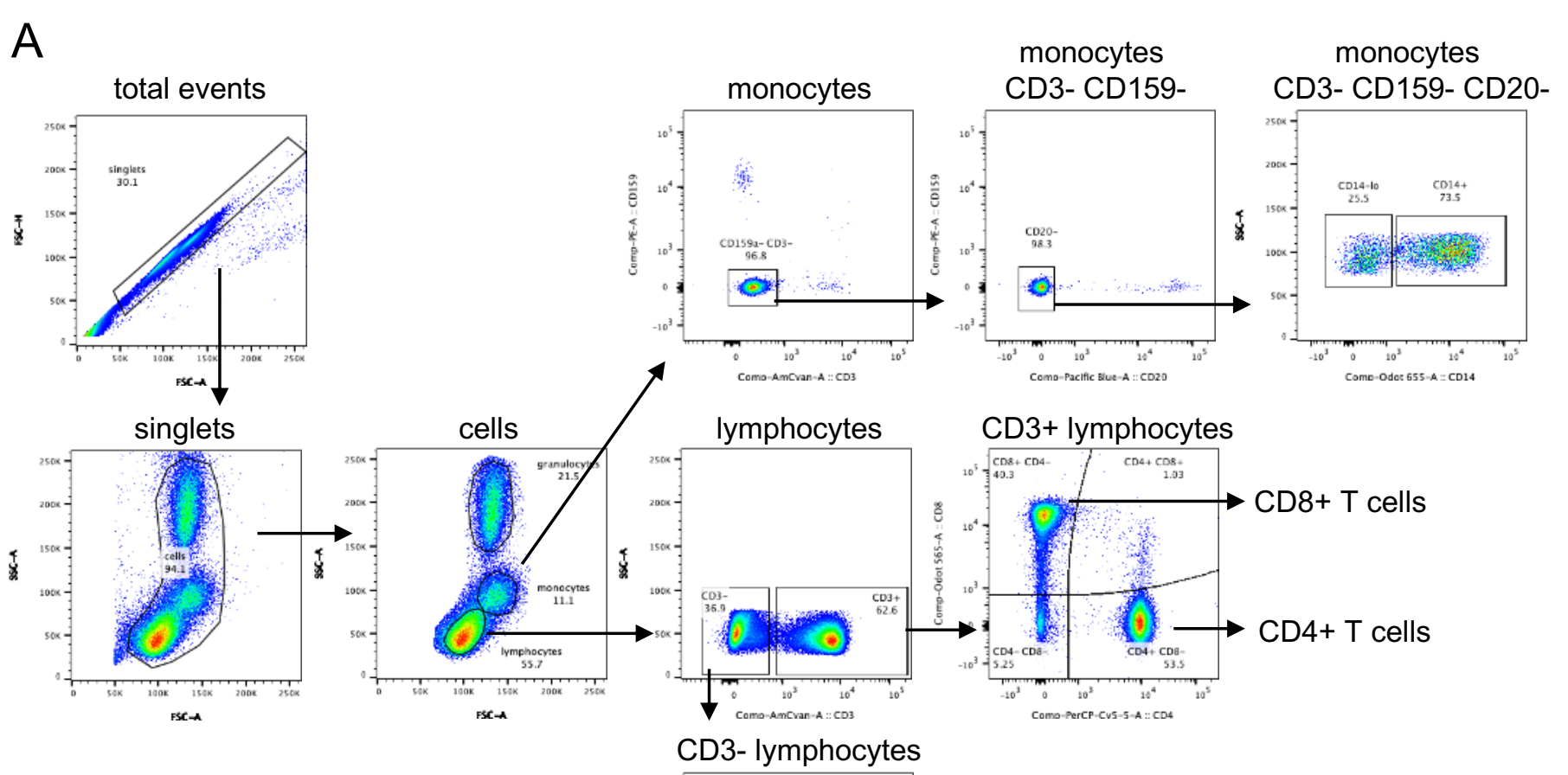

Come-odot 655-A: CD14

(1)

